\title{
Forschungsberichte
}

\section{Struktur Subjektiver Theorien über Musikalität und Validierung eines Messinstruments}

\author{
Structure of Theory of Musicality and Validation of a Measuring Instrument
}

\author{
Miriam Eisinger ${ }^{*_{1}}$
}

[1] Institut für Musik, Pädagogische Hochschule Freiburg, Freiburg, Deutschland.

\section{Zusammenfassung}

Musiker*innen werden geboren, nicht gemacht. Diese Überzeugung findet sich häufig in der allgemeinen Bevölkerung, bei vielen Musiker*innen und auch Musiklehrpersonen. Im Rahmen dieser Überzeugung wird Musikalität als angeboren und unveränderbar angesehen. Solch eine stabile Subjektive Theorie über Musikalität steht im Gegensatz zu einer dynamischen Subjektiven Theorie über Musikalität, bei der musikalische Fähigkeiten als veränderbar und erlernbar betrachtet werden. Eine dynamische Subjektive Theorie über Musikalität ist jedoch ein entscheidender Faktor dafür, dass Personen ihr musikalisches Potential entfalten können. Um die Subjektiven Theorien über Musikalität empirisch erfassen zu können, war das Ziel der vorliegenden Studie die Struktur der Subjektiven Theorien in der Domäne Musik mithilfe eines englisch- und eines deutschsprachigen Messinstruments zu überprüfen. Dafür wurde ein für den Musikbereich adaptiertes Selbstauskunftsinventar von englischen $(n=595,71,6 \%$ weiblich, $M=13,49, S D=1,74)$ und deutschen $(n=$ $385,53,2 \%$ weiblich, $M=10,51$, SD = 0,71) Schüler*innen ausgefüllt. Die Ergebnisse der Reliabilitätsüberprüfungen und der konfirmatorischen Faktorenanalyse sprechen für ein multidimensionales, hierarchisches Konstrukt, welches zwei Faktoren zweiter Ordnung enthält (Entity und Incremental), und weiter differenziert zwischen der Ursache und der Veränderbarkeit von Musikalität mit vier Faktoren erster Ordnung (Learning vs. Gift und Improvement vs. Stable). Durch das Messinstrument können bei zukünftigen (Langzeit-) Untersuchungen individuelle Unterschiede in der Entwicklung musikalischer Subjektiver Theorien von Schüler*innen aufgedeckt und der Einfluss der Subjektiven Theorien über Musikalität auf die allgemeine musikalische Entwicklung untersucht werden.

Schlüsse/wörter: Subjektive Theorien, Musikalität, Schüler*innen, Fragebogen, Validierung, Motivation, Einstellung

\begin{abstract}
Musicians are born, not made. This belief is held by a majority of the general population, musicians and music teachers. In the framework of this belief musicality is seen as stable and inherent. This entity view of the Implicit Theory of Musicality contrasts with the incremental view in which musical abilities are seen as malleable and learnable. However, an incremental Implicit Theory of Musicality is an important prerequisite for the development of individual musical potentials. In order to be able to asses these Implicit Theories of Musicality, the aim of the present study was to validate the structure of Implicit Theories in the domain of music with an English and German version of a standardised measurement instrument. Therefore English $(n=595,71.6 \%$ female, $M=13.49, \mathrm{SD}=1.74)$ and German $(n=385,53.2 \%$ female, $M=10.51, S D=0.71$ ) students completed a self-report inventory, which was adapted for the music domain. The results of the reliability analysis and the confirmatory factor analysis suggest a multidimensional, hierarchical construct, which contains two second-orderfactors (Entity and Incremental) and distinguishes between the origin and the stability of musicality with four first-order-factors (Learning vs. Gift and Improvement vs. Stable). The new measurement instrument allows to quantify individual differences in the attitudes towards one own's musical ability and determine the importance of Implicit Theories of Musicality for the general development of musical abilities.
\end{abstract}

Keywords: implicit theories, musicality, students, questionnaire, validation, motivation, attitude

Jahrbuch Musikpsychologie, 2021, Vol. 30: Musikpsychologie - Empirische Forschungen - Ästhetische Experimente, Artikel e93, https://doi.org/10.5964/jbdgm.93

Eingereicht: 2021-01-27. Akzeptiert: 2021-07-14. Publiziert (VoR): 2021-08-17.

Begutachtet von: Andreas Heye; Anna Wolf.

*Korrespondenzanschrift: Institut für Musik, Pädagogische Hochschule Freiburg, Kunzenweg 21, 79117 Freiburg, Deutschland. E-Mail: miriam.eisinger@ph-freiburg.de

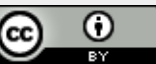

Dieser Open-Access-Artikel steht unter den Bedingungen einer Creative Commons Namensnennung 4.0 International Lizenz, CC

BY 4.0 (https://creativecommons.org/licenses/by/4.0/deed.de). Diese erlaubt für beliebige Zwecke (auch kommerzielle) den Artikel zu verbreiten, in jedwedem Medium zu vervielfältigen, Abwandlungen und Bearbeitungen anzufertigen, unter der Voraussetzung, dass der Originalartikel angemessen zitiert wird. 


\section{Einleitung und theoretischer Hintergrund}

Von Musiklehrkräften, Musiker*innen, aber auch der allgemeinen Bevölkerung werden Musikalität oder auch musikalische Begabung häufig als unveränderbar oder ausschließlich genetisch bedingt angesehen (Austin et al., 2006; Sloboda et al., 1994). Dieses Alltagsverständnis steht im Gegensatz zu Ergebnissen wissenschaftlicher Untersuchungen, die zeigen, dass individuelle Unterschiede in der musikalischen Entwicklung von Jugendlichen durch ein Zusammenspiel von Anlage- und Umweltfaktoren hervorgerufen werden. Solche Umweltfaktoren können beispielsweise die Unterstützung durch die Eltern oder auch das Handeln von Lehrpersonen sein (Hallam, 2006; Howe et al., 1998; McPherson \& Williamon, 2006; Sloboda et al., 1994).

Neben empirischen Untersuchungen zu dieser Anlage-Umwelt-Debatte hinsichtlich Musikalität sind die Überzeugungen über die Beschaffenheit von Musikalität selbst Gegenstand von Forschung. Die Forschungen hierzu basieren vor allem auf den Untersuchungen von Dweck $(1986,2000)$ zu Implicit Theories, welche von ihr definiert werden als „core assumptions about the malleability of personal qualities” (Yeager \& Dweck, 2012, S. 303). Im Rahmen dieser Theorie unterscheiden sich Personen darin, ob sie persönliche Eigenschaften wie die Intelligenz als unveränderbar bzw. stabil ansehen oder die Überzeugung haben, dass diese Eigenschaften sich z. B. durch Anstrengungen entwickeln können und damit veränderbar, also dynamisch, sind (Dweck \& Grant, 2008).

In bisherigen Studien fehlt jedoch die Validierung eines deutsch- und englischsprachigen Messinstruments zur Erfassung dieser Implicit Theories in der Domäne Musik.

\section{Klärung der Begrifflichkeiten}

Ein Überblick über den Diskurs zu den Implicit Theories zeigt allerdings, dass sowohl Dweck selbst als auch weitere Autor*innen in ihren Veröffentlichungen jeweils unterschiedliche Begrifflichkeiten für dieses Konstrukt verwenden. Dabei werden diese Begriffe häufig synonym verwendet und in ihren Definitionen nicht klar voneinander abgegrenzt (Lüftenegger \& Chen, 2017). In der englischsprachigen Literatur werden Begriffe wie Implicit Theories (u. a. Dweck et al., 1995; Wang et al., 2018), Mindsets (u. a. Adams, 2019; Burnette et al., 2013; Dweck, 2008), Self-Theories (u. a. Dweck, 2000; Müllensiefen et al., 2015; O’Neill, 2011) oder auch Lay Theories (u. a. Biddle et al., 2003; Molden \& Dweck, 2006) verwendet und in der deutschsprachigen Literatur sind u. a. die Begriffe Selbstbild (u. a. Dweck, 2017), Subjektive Theorien (u. a. Rieche et al., 2018; Woolfolk, 2008), Subjektive Überzeugungen (u. a. Spinath \& Schöne, 2003) und Implizite Theorien (u. a. Spinath, 2001) zu finden. In der vorliegenden Studie wird der Begriff Subjektive Theorien verwendet, um hervorzuheben, dass Subjektive Theorien analog zu wissenschaftlichen Theorien ein Bezugssystem für das eigene Denken und Handeln bilden, sich jedoch im Gegensatz zu diesen aus alläglichen, subjektiven Erfahrungen einer Person zusammensetzen (Yeager \& Dweck, 2012).

Obwohl es Uneinigkeit darüber gibt, wie kontextspezifisch Subjektive Theorien sind (Lüftenegger \& Chen, 2017), zeigen Studien, dass Personen unterschiedliche Theorien für verschiedenen Eigenschaften besitzen können (Dweck \& Grant, 2008). Deshalb müssen Subjektiven Theorien in verschiedenen Domänen mit domänenspezifischen Instrumenten gemessen werden (Dweck, 2000). Ausgehend von der Forschung von Dweck 
wurden die Theories of Intelligence auf viele weitere Domänen wie die Persönlichkeit, Führungsqualitäten oder auch sportliche Fähigkeiten übertragen (Burnette et al., 2013).

Für die Domäne Musik wird in der vorliegenden Studie analog zu dem Begriff Intelligenz bei Dweck (2000) von Musikalität gesprochen. Auch hier herrscht jedoch keine begriffliche Klarheit, denn in Veröffentlichungen zu Subjektiven Theorien in der Domäne Musik werden nicht nur die Begriffe Musikalität (Rieche et al., 2018) bzw. Musicality (Müllensiefen et al., 2015) verwendet, sondern z. B. auch Musical Ability (Austin et al., 2006; O'Neill, 2011; Smith, 2005), und Beliefs in Music (Wang et al., 2018). In akademischen Kontexten herrscht kein Konsens darüber, was mit dem Begriff Musikalität gemeint ist (Hallam, 2006). Unter Berücksichtigung dieser Uneinigkeit schlägt Gembris (2018) folgende Arbeitsdefinition vor: „Musikalität ist die allgemeine, angeborene und universelle menschliche Disposition zur Kommunikation mit gestalteten Tönen, Rhythmen und Klängen“ (Gembris, 2018, S. 236). Während Begriffe wie musikalische Begabung, Talent oder musikalische Fähigkeiten jeweils einen Schwerpunkt auf Anlage- bzw. Umweltbedingungen implizieren und verschiedene Bedeutungen in englischer und deutscher Sprache haben können, ist Musikalität in erster Hinsicht die neutralste Form und ist die wörtliche Übersetzung zu Musicality (Hemming, 2002). Deshalb wird im Kontext dieser Studie die Bezeichnung Subjektive Theorien über Musikalität verwendet und synonym zu den oben genannten Begriffen verstanden.

\section{Die Subjektiven Theorien über Musikalität als ein entscheidender Faktor in Motivationsprozessen}

Subjektive Theorien sind ein zentraler Bestandteil von pädagogischen und auch musikalischen Motivationsmodellen (Hargreaves \& Lamont, 2017; Helmke, 2017). Basierend auf Connell (1990) entwickelten Austin et al. (2006) ein solches theoretisches Motivationsmodell. In diesem beschreiben die Autor*innen, wie die Dimensionen Self System, Social System, Outcomes und Actions sich wechselseitig beeinflussen und eine optimale Motivation für das Lernen in musikalischen Kontexten entstehen kann (siehe Abbildung 1). Dieses Motivationssystem entwickelt und verändert sich im Laufe der Zeit durch Lernprozesse und vor allem durch die Herausforderungen, die den Lernenden begegnen. Den Subjektiven Theorien über Musikalität kommt als Teil des Self System in diesem Modell eine wesentliche Rolle zu, da sie den Rahmen vorgeben, wie Schüler*innen ihre musikalischen Erfahrungen interpretieren und wie diese ihre Aussichten auf zukünftige musikalische Lernerfolge einschätzen (Austin et al., 2006). Weitere Aspekte des Self Systems sind u. a. das Musikalische Selbstkonzept und die Attribution von musikalischen Misserfolgen. Im Gegensatz zu den Subjektiven Theorien über Musikalität beschreibt das Konstrukt des Musikalischen Selbstkonzepts nicht das Konzept einer Person hinsichtlich der Eigenschaften von Musikalität, sondern „Wer ich bin und was ich kann in der Musik" (Spychiger, 2013, S. 18). Zudem beschreiben die Subjektiven Theorien den übergeordneten Bedeutungsrahmen, in welchem verschiedene Ursachenzuschreibungen und Erklärungsmöglichkeiten für Misserfolge und Erfolge in Musik auftreten. Sie können z. B. erklären, wie Personen überhaupt in solche Situationen gelangen (Dweck, 2000). Somit können die Subjektiven Theorien über Musikalität von diesen und anderen Aspekten des Self Systems abgegrenzt werden. 


\section{Abbildung 1}

Modell der optimalen Motivation für Lernen in musikalischen Kontexten (Austin et al., 2006, S. 232)

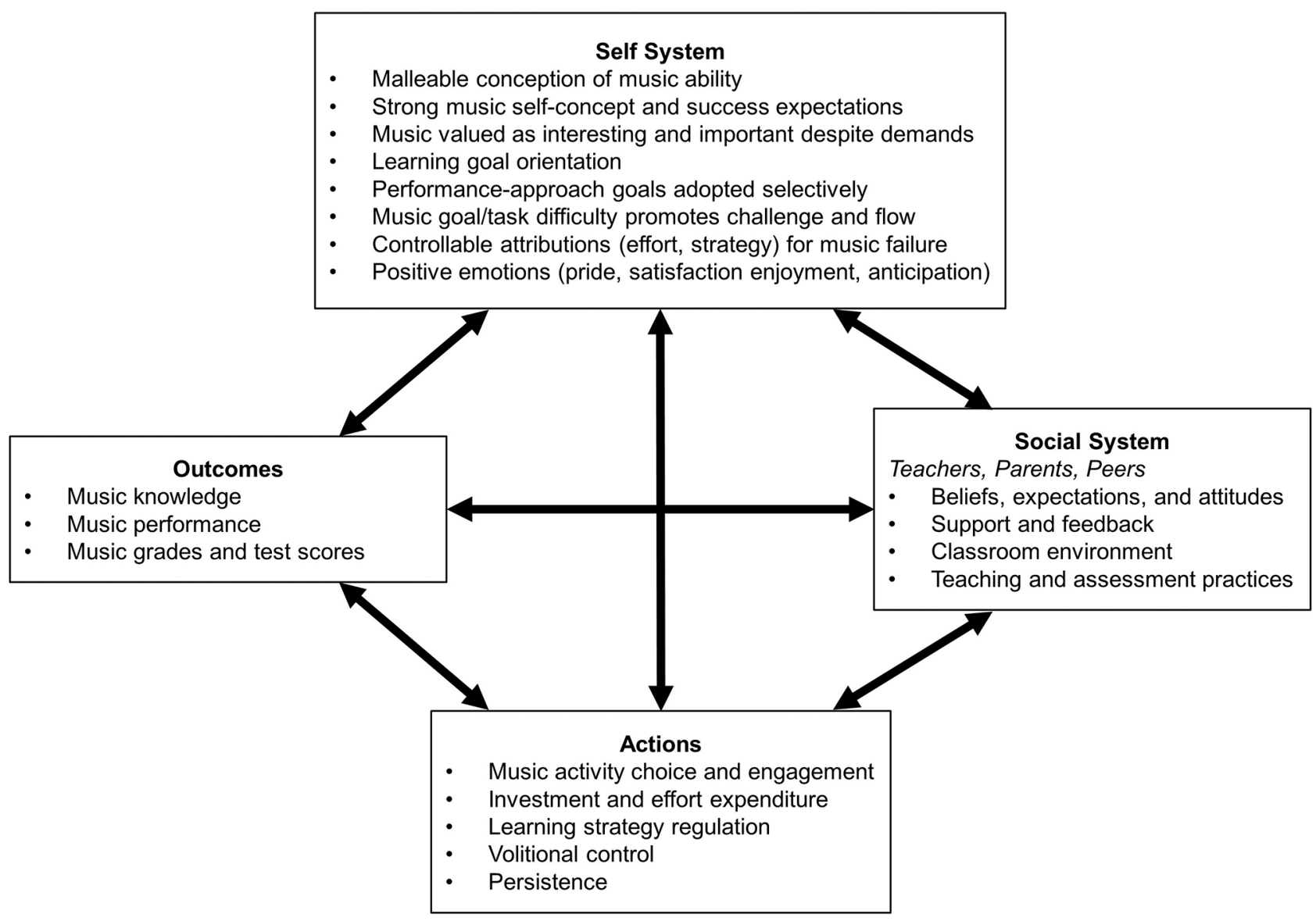

Die theoretischen Annahmen zu der Wirkung von Subjektiven Theorien über Musikalität können von Studienergebnissen gestützt werden, die u. a. einen positiven Zusammenhang zwischen einer dynamischen Subjektiven Theorie über Musikalität und den allgemeinen schulischen Leistungen, der musikalischen Aktivität und spezifischen musikbezogenen Wahrnehmungsfähigkeiten aufzeigen (Müllensiefen et al., 2015). Allgemein zeigen viele Forschungen zu den Subjektiven Theorien, dass Personen vor allem angesichts von Herausforderungen oder drohendem Misserfolg unterschiedliche Reaktionen zeigen, je nachdem, welche Subjektive Theorie sie besitzen (Dweck, 2000). So zeigen auch Studien in musikbezogenen Kontexten, dass Kinder mit einer stabilen Subjektiven Theorie über Musikalität dazu tendieren, sich hilflos angesichts von Misserfolgen beim musikalischen Lernen zu verhalten. Kinder mit einer dynamischen Subjektiven Theorie über Musikalität überlegen im Gegensatz dazu in solchen Situationen möglichen Versagens beispielsweise, welche Strategien oder Anstrengungsveränderungen notwendig sind, um zukünftig Erfolg zu haben (O'Neill, 2011; O'Neill \& Sloboda, 1997).

Die große Bedeutung von Subjektiven Theorien bei der Bewältigung von Herausforderungen bzw. Misserfolgen zeigt die hohe Relevanz auf, die die Erforschung Subjektiver Theorien in musikalischen Kontexten hat. Denn der Weg zu hoher musikalischer Leistung wird oft durch viele Hindernisse begleitet, für deren Bewältigung entsprechende Motivation und Einstellungen vorhanden sein müssen (Hargreaves \& Lamont, 2017; MacNamara et al., 2006; O'Neill, 2011). Zudem entstehen in schulischen Kontexten im Fach Musik viele Gelegenheiten, bei 
denen die Unterschiede zwischen den Peers in den musikalischen Fähigkeiten offensichtlich werden. Die Performanz bei Schulaufführungen oder auch beim Klassenvorspiel birgt immer auch die Gefahr von öffentlichem Versagen (Austin et al., 2006). Somit ist es notwendig, sich mit den Subjektiven Theorien auch im Kontext von Musiklernen bzw. Musikunterricht zu beschäftigen

\section{Unklarheiten bei Struktur und Messung Subjektiver Theorien}

In der Forschung über Subjektive Theorien herrscht jedoch über die verschiedenen Domänen hinweg Uneinigkeit darüber, ob diese als ein- oder mehrdimensionales Konstrukt aufgefasst werden müssen (Lüftenegger \& Chen, 2017). Dweck (2000) nutzt zu Beginn ihrer Forschung einen Fragebogen, der nur Aussagen zu einer stabilen Subjektiven Theorie über Intelligenz enthält. Aus den Antworten wird dann bei der Datenanalyse eine Gruppe von stabilen Theoretiker*innen (hohe Zustimmungswerte) und dynamischen Theoretiker*innen (niedrige Zustimmungswerte) gebildet, womit die beiden Subjektiven Theorien als gegensätzliche Pole einer Skala angesehen werden (Dweck, 2000). Diese Version des Fragebogens wurde für verschiedenste weitere Domänen adaptiert und eine eindimensionale bipolare Skala wird heute noch von der großen Mehrheit der empirischen Forschung verwendet (Burnette et al., 2013).

Es scheint jedoch auch möglich, dass Personen beide Subjektive Theorien vertreten, und ein stark negativer Zusammenhang zwischen der stabilen und der dynamischen Subjektiven Theorie kann nicht eindeutig von Studien nachgewiesen werden (Lüftenegger \& Chen, 2017). Zudem zeigt ein Modellvergleich von Tempelaar et al. (2015) anhand der Theories of Intelligence Scale - Self Form for Adults (Dweck, 2000) und mithilfe einer Stichprobe von Studierenden einen besseren Model Fit für ein 2-Faktoren-Modell im Vergleich zu einem bipolaren 1-Faktor-Modell. Nach Lüftenegger und Chen (2017) sprechen deshalb viele Gründe gegen die Vorstellung, dass eine stabile Theorie und eine dynamische Theorie buchstäblich das Gegenteil voneinander sind. Es fehlen jedoch diesbezüglich empirische Studien, weshalb sie weitere Untersuchungen hinsichtlich der Struktur der Subjektiven Theorien fordern (Lüftenegger \& Chen, 2017). Auch in der Domäne Musik werden die Subjektiven Theorien nicht einheitlich operationalisiert. So erfassen z. B. Müllensiefen et al. (2015) die Subjektiven Theorien über Musikalität eindimensional, während Smith (2005) und Wang et al. (2018) in einem englischsprachigen und Rieche et al. (2018) in einem deutschsprachigen Messinstrument die Subjektiven Theorien auf zwei Dimensionen erfassen. Es werden demnach empirische Studien benötigt, die untersuchen, inwiefern die Subjektiven Theorien über Musikalität als ein- oder mehrdimensionales Konstrukt aufgefasst werden können.

Für die Domäne Sport überprüften Biddle et al. (2003) die Struktur der Subjektiven Theorien mithilfe eines Modellvergleichs, wobei die Autor*innen nicht nur zwischen den beiden Faktoren dynamische Subjektive Theorie (Incremental) und stabile Subjektive Theorie (Entity) unterscheiden, sondern zusätzlich in den Überzeugungen über die Ursache (Learning vs. Gift) und die Stabilität (Improvement vs. Stable) von sportlichen Fähigkeiten. Die konfirmatorische Faktorenanalyse spricht für ein hierarchisches Modell 2. Ordnung, wobei der Faktor Incremental von den untergeordneten Faktoren Learning und Improvement und der Faktor Entity von den untergeordneten Faktoren Gift und Stable gebildet wird. Wang et al. (2018) adaptierten diesen Fragebogen für die Bereiche Musik und Kunst, und konnten das angenommene hierarchische Modell, angelehnt an Biddle et al. (2003), empirisch belegen. Dabei verglichen sie jedoch nur das angenommene hierarchische Modell mit den empirischen Daten, statt die Ein- bzw. Mehrdimensionalität der Subjektiven Theorien über Musikalität anhand von unterschiedlichen Modellen zu überprüfen. Die Autor*innen stellten zudem fest, dass eine Verallge- 
meinerung der Ergebnisse in z. B. europäische Kontexte limitiert sein könnte, da in der für diese Analysen verwendeten Stichprobe die Teilnehmenden alle dem gleichen Lehrerausbildungsinstitut in Singapur zugehörig waren. Für die Untersuchung wurde außerdem eine kleine Stichprobe von $n=103$ Studierenden mit einem Altersdurchschnitt von $M=26,98$ Jahren $(S D=4,79)$ verwendet (Wang et al., 2018). Studien geben jedoch Hinweise darauf, dass die Struktur der Subjektiven Theorien sich im Laufe des Heranwachsens einer Person verändert und es gerade an der weiterführenden Schule zu solchen Strukturveränderungen kommen kann (Dweck, 2000; Sarrazin et al., 1996).

Zusammenfassend fehlen deshalb bisher Studien, die einerseits mithilfe eines Modellvergleichs die Subjektiven Theorien über Musikalität in einer englischsprachigen, europäischen Stichprobe mit Schüler*innen validieren und andererseits die Struktur der Subjektiven Theorien über Musikalität in einem deutschsprachigen Fragebogen untersuchen.

\section{Zielsetzung und Fragestellungen}

Das Ziel der vorliegenden Studie ist, eine deutsche und englische Version des Fragebogens zur Erfassung der Subjektiven Theorien über Musikalität zu validieren und mithilfe dieses Messinstruments die Struktur dieses Konstrukts zu überprüfen. Dabei soll die Trennung der beiden Dimensionen dynamische Subjektive Theorie über Musikalität (Incremental) und stabile Subjektive Theorie über Musikalität (Entity) empirisch untersucht werden.

Folgende Forschungsfragen werden formuliert:

- Zeigt das hierarchische Model der Subjektiven Theorien über Musikalität analog zu dem Messinstrument der Subjektiven Theorien über sportliche Fähigkeiten im Vergleich mit anderen Strukturmodellen den besten Modell Fit?

- Können für die latenten Variablen dieses Modells reliable Skalen gebildet werden?

\section{Methodisches Vorgehen}

\section{Stichprobe}

Die Stichprobe wurde entnommen aus drei Erhebungswellen der Befragung der Schüler*innen im Rahmen einer Langzeitstudie (LongGold-Projekt) im Zeitraum von 2016 bis 2018. In dieser musikpsychologischen Studie werden seit 2015 mithilfe eines Kohorten-Sequenz-Designs (Prinzie \& Onghena, 2005) die Entwicklung von musikalischen Kompetenzen im Zusammenhang mit kognitiven und sozialen Fähigkeiten, Überzeugungen und musikbezogenen Aktivitäten bei Schüler*innen von der fünften bis zur zehnten Klasse der allgemeinbildenden Schule in England und Deutschland untersucht. Für die Stichprobe der vorliegenden Studie wurden nur die Schüler*innen miteinbezogen, die zu dem jeweiligen Erhebungszeitpunkt das erste Mal an der Langzeitstudie teilnahmen, um eine Doppelung der Fälle im Datensatz zu vermeiden.

Der so gewonnene Datensatz der vorliegenden Studie umfasst die Daten von 595 englischsprachigen Schüler*innen (71,6\% weiblich) im Alter von 10 bis 18 Jahren $(M=13,49, S D=1,74)$ und 385 deutschsprachige Schüler*innen (53,2\% weiblich) im Alter von 9 bis 13 Jahren $(M=10,51, S D=0,71)$. Die ungleiche Geschlech- 
terverteilung in der englischen Stichprobe resultiert daraus, dass ein großer Teil der Teilnehmenden dieser Stichprobe einer Mädchenschule in Südengland zugehörig war $(n=359)$. Insgesamt waren drei englische und fünf deutsche Schulen an der vorliegenden Studie beteiligt. Da Studien (Dweck, 2000; Hallam, 2006; Sloboda et al., 1994) Hinweise auf kulturelle Unterschiede sowohl hinsichtlich der Subjektiven Theorien, als auch im Verständnis von Musikalität, geben, wurden die englische und deutsche Stichprobe getrennt voneinander analysiert.

\section{Erhebungsinstrumente}

Um ein geeignetes englisches und deutsches Messinstrument zur Überprüfung der Struktur der Subjektiven Theorien über Musikalität zur Verfügung zu haben, wurde ein neues Messinstrument entwickelt, welches auf dem Fragebogen zu den Subjektiven Theorien über sportliche Fähigkeiten (Biddle et al., 2003) basiert und seit 2016 bei den Erhebungen des LongGold-Projekts verwendet wurde. Die 12 Items des englischen Fragebogens von Biddle et al. (2003) wurden für den musikalischen Kontext adaptiert, indem das Wort „sport" durch „music" ausgetauscht wurde, was dem Vorgehen von Wang et al. entspricht, wie diese Autoren es in ihrer Veröffentlichung 2018 beschreiben. Zusätzlich wurde bei dem von Mitarbeitenden des LongGold-Projekts entwickelten Fragebogens bei dem Item mit der Item-Kennung STM_2 der Zusatz „on your instrument“ ergänzt (Items siehe Tabelle A1 im Anhang). Der Grad der Zustimmung zu den Aussagen wird auf einer fünfstufigen Likert-Skala von 1 (strongly disagree) bis 5 (strongly agree) angegeben.

Die englischen Items des Fragebogens wurden dann von Mitarbeitenden des LongGold-Projekts in die deutsche Sprache übersetzt, um den Fragebogen auch in den deutschen Standorten des LongGold-Projekts verwenden zu können. Hinsichtlich der Übersetzung bestand folgende Herausforderung: Dweck (2000) unterscheidet bei den Messinstrumenten zu den Subjektiven Theorien zwischen einer „Self“-Form und einer „Other"Form. Die „Self“-Form fragt die Personen nach deren Subjektiven Theorie zu ihren eigenen, persönlichen Eigenschaften, wie z. B. in folgendem Item: „You have a certain amount of intelligence ..." (Dweck, 2000, S. 177). Die „Other“-Form bezieht sich auf die Eigenschaft im Allgemein, wie z. B. „Everyone has a certain amount of intelligence ..." (Dweck, 2000, S. 175). Jedoch kann das englische Wort „You“ nicht nur mit "Du“, sondern auch mit „Man“ übersetzt werden (Oxford University Press, 2020). Das heißt durch die englischen Items wird nicht deutlich, ob sich die Aussagen auf die Subjektive Theorie zu der eigenen Intelligenz oder zu der Intelligenz im Allgemeinen beziehen. Bei Biddle et al. (2003) wird jedoch sowohl in der Beschreibung des Messinstruments, als auch in dem Einleitungstext zu den Items des Fragebogens nicht dargestellt, dass mit dem Fragebogen nur die Subjektiven Theorien in Bezug auf die eigenen sportlichen Fähigkeiten erfasst werden sollen. Deshalb wurde bei der Übersetzung des adaptierten Fragebogens zu den Subjektiven Theorien über Musikalität die Ansprache „You“, wie z. B. in dem Item „You have a certain level of musical ability and you cannot really do much to change it“ (Item-Kennung STM_1) mit dem Wort „Man“ übersetzt, d. h. für das Beispielitem: „Man hat ein gewisses Level an musikalischen Fähigkeiten und kann nicht viel tun, um das zu ändern" (siehe Tabelle A1 im Anhang).

Die Übersetzung des englischen Fragebogens in die deutsche Sprache wurde in einem iterativen Verfahren von Mitarbeitenden des LongGold-Projekts überarbeitet und in einer kleinen Pilotstichprobe von deutschen Schüler*innen der fünften Klasse $(n=20)$ überprüft. In die Überarbeitung der Items wurden zudem die Rückmeldungen von Lehrpersonen hinsichtlich der Verständlichkeit der Aussagen für Schüler*innen mitaufgenommen. Um die Übersetzung des englischen Fragebogens in die deutsche Version zusätzlich zu validieren, wurde 
die englische und deutsche Fassung der Items drei unabhängigen bilingualen Personen von der Autorin dieses Beitrags zur Überprüfung vorgelegt. Ziel des Vergleichs der Items war neben der allgemeinen Überprüfung der Übersetzung vor allem die Übersetzung des Wortes „You“ mit „Man“ zu validieren. Dieses Anliegen war den Expert*innen unbekannt. Da keine der Personen die Übersetzung des Wortes „You“ kritisierte und sich aus den wenigen Kommentaren zu den Items keine einheitlichen, schwerwiegenden Änderungsnotwendigkeiten ergaben, wurden die Items des Fragebogens nachträglich nicht mehr verändert. Eine Auflistung der Items des englischsprachigen und des deutschsprachigen Fragebogens zur Erfassung der Subjektiven Theorien über Musikalität ist im Anhang (Tabelle A1) zu finden.

Für die vorliegende Studie wurden bei der Datenerhebung zusätzlich das Alter, Geschlecht, die Nationalität und Klassenstufe der Schüler*innen erhoben.

\section{Datenerhebung}

Die Erhebung erfolgte im Rahmen der Langzeitstudie LongGold in den Jahren 2016 bis 2018 und wurde unter Aufsicht von Mitarbeitenden des LongGold-Projekts und einer Lehrkraft in der Schule mithilfe eines Online-Fragebogens an Tablets bzw. Computern durchgeführt. Den Schüler*innen standen dabei 90 Minuten zur Bearbeitung der gesamten Testbatterie inklusive der Fragebögen für die vorliegende Studie zur Verfügung. Für die Bearbeitung der 12 Items des Fragebogens zur Erfassung der Subjektiven Theorien über Musikalität benötigten die Schüler*innen 3 - 6 Minuten. Die Studie wurde von den Ethikkommissionen des Goldsmiths College, der Leibniz Universität Hannover und dem Ministerium für Kultus, Jugend und Sport Baden-Württemberg genehmigt und nach Einverständnis der beteiligten Schulen und der Erziehungsberechtigten durchgeführt. Die Teilnahme an dem LongGold-Projekt ist für die Schüler*innen freiwillig und das Einverständnis kann zu jedem Zeitpunkt widerrufen werden. Um individuelle Veränderungen der erhobenen psychometrischen Konstrukte bei den Schüler*innen über den Untersuchungszeitraum der Langzeitstudie nachvollziehen zu können, werden die Daten der Schüler*innen mithilfe einer 14-stelligen Identifikationsnummer pseudoanonymisiert. Die Zuordnung von Identifikationsnummer und Klarnamen der Schüler*innen ist dabei nur den Verantwortlichen der Schule möglich.

\section{Datenanalyse}

Um festzustellen, ob die empirischen Daten die angenommene Struktur der Subjektiven Theorien über Musikalität unterstützen (Fragestellung 1), wurde nach der Datenbereinigung eine konfirmatorische Faktorenanalyse (KFA) berechnet. Da für diese Analysen formal die Voraussetzung von multivariat normalverteilten Indikatorvariablen besteht (Döring \& Bortz, 2016, S. 957), wurde diese Normalverteilung mithilfe des Mardia's-Tests überprüft. Zur Analyse der Daten wurden das Lavaan Paket (Rosseel, 2012) und das MVN Paket (Korkmaz et al., 2014) der Statistik Software $R$ (R Core Team, 2020) verwendet. Zunächst wurde ein eindimensionales Modell (M0) in die KFA miteinbezogen. Zudem wurden analog zu Biddle et al. (2003) folgende Modelle miteinander verglichen: Modell 1 (M1) mit den beiden latenten Faktoren Incremental und Entity, Modell 2 (M2) testet vier Faktoren erster Ordnung (Learning, Improvement, Gift, Stable) und Modell 3 (M3) testet ein hierarchisches Modell, bei dem die beiden untergeordneten Faktoren Stable und Gift auf dem übergeordneten Faktor Entity basieren und der Faktor Incremental die beiden untergeordneten Faktoren Learning und Improvement enthält. Alle angenommenen Messmodelle sind in Abbildung 2, 3, 4 und 5 dargestellt. 


\section{Abbildung 2}

Struktur des eindimensionalen Modells (MO)

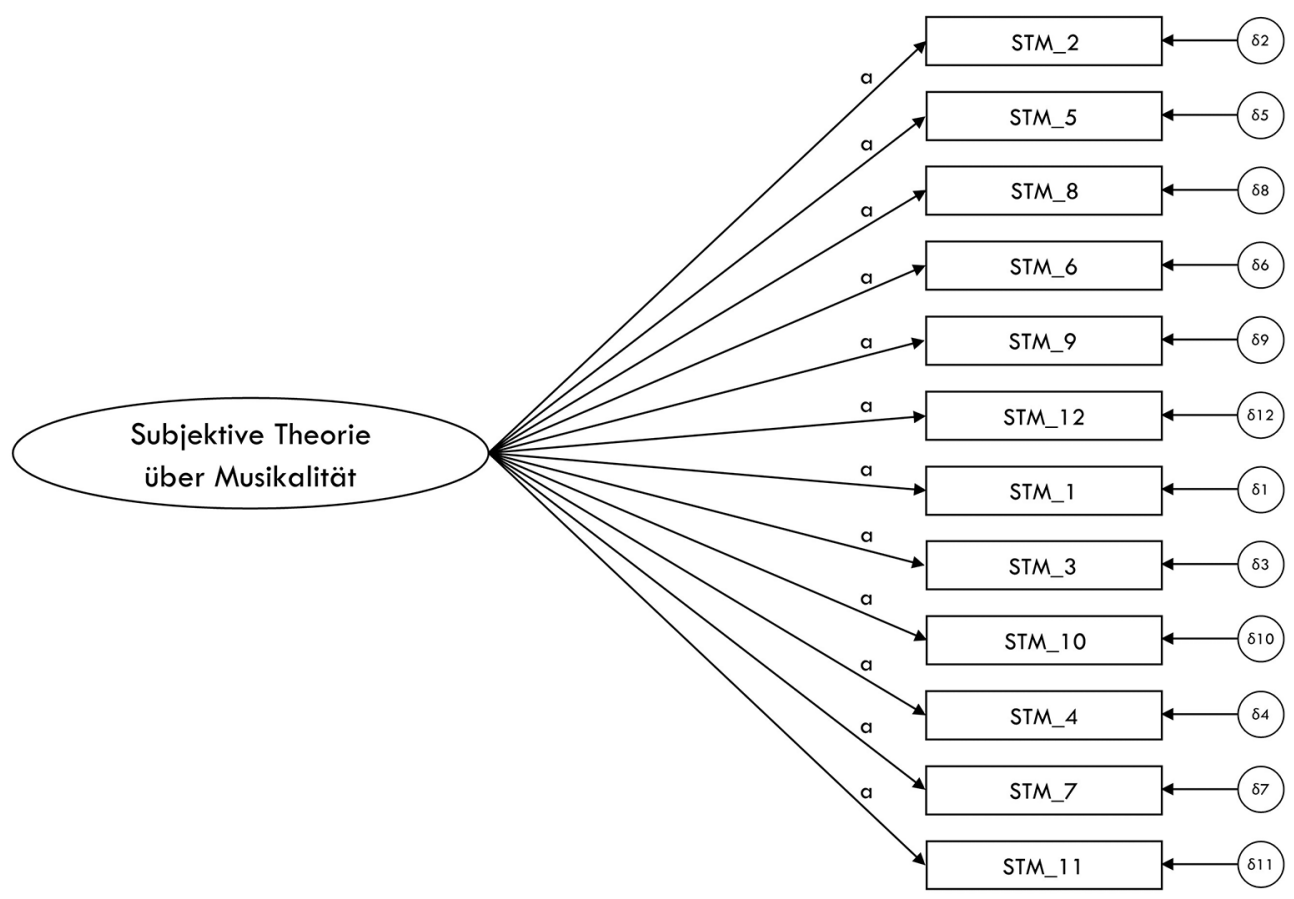

Anmerkung. STM_X = Item-Kennung.

\section{Abbildung 3}

Struktur des zweidimensionalen Modells (M1)

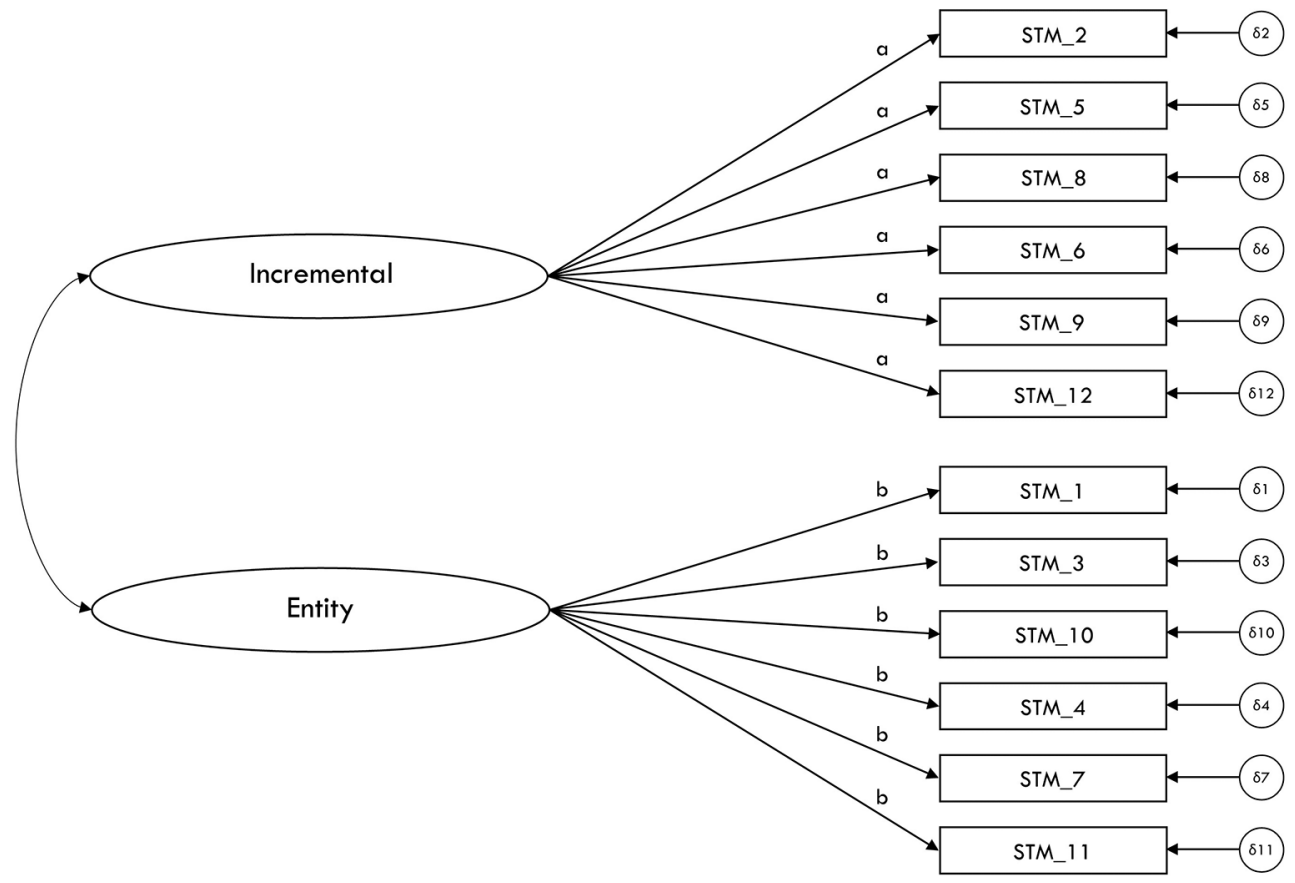

Anmerkung. STM_X = Item-Kennung. 


\section{Abbildung 4}

Struktur des vierdimensionalen Modells (M2)
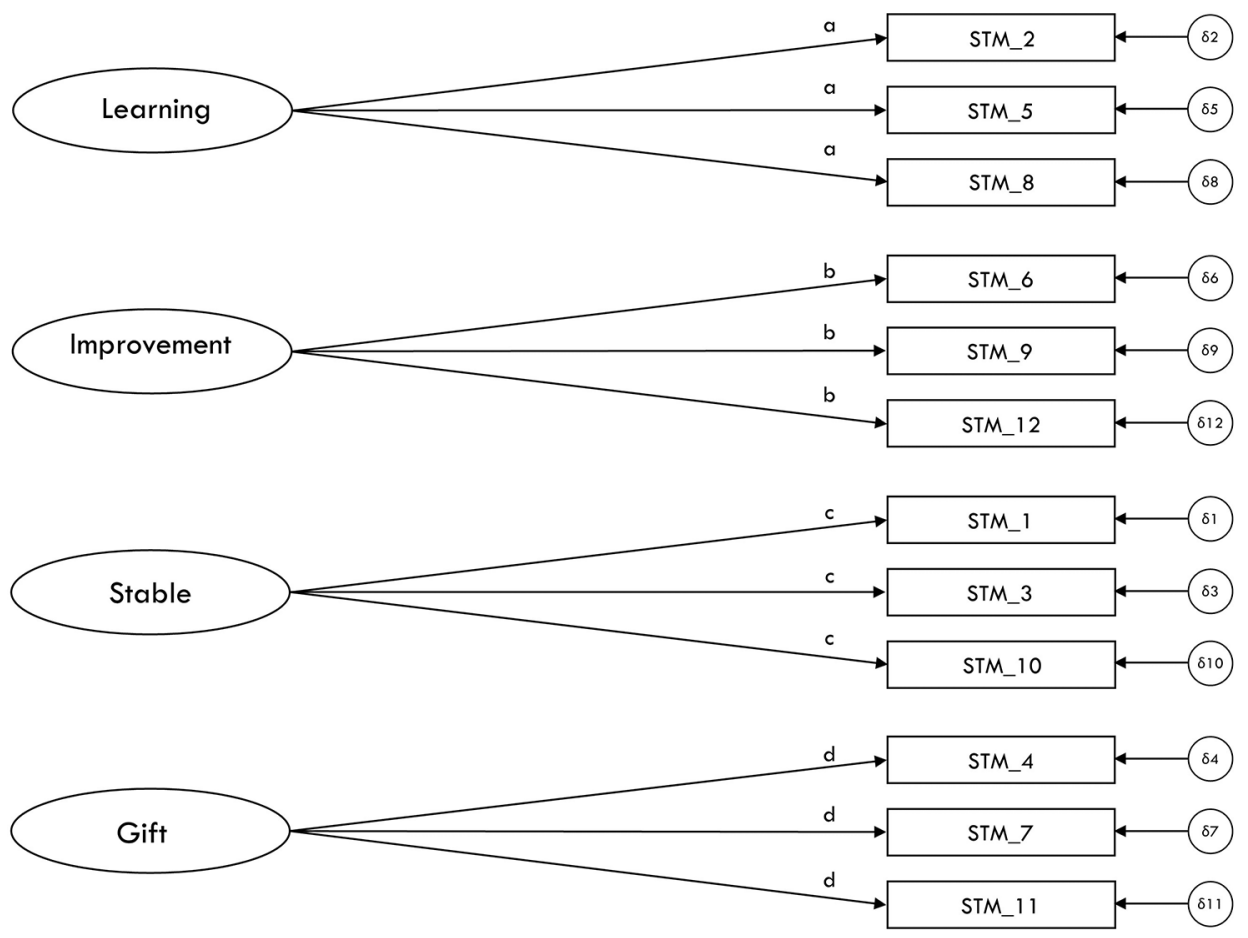

Anmerkung. STM_X = Item-Kennung.

\section{Abbildung 5}

Struktur des hierarchischen Modells mit vier Faktoren erster Ordnung und zwei Faktoren zweiter Ordnung (M3)

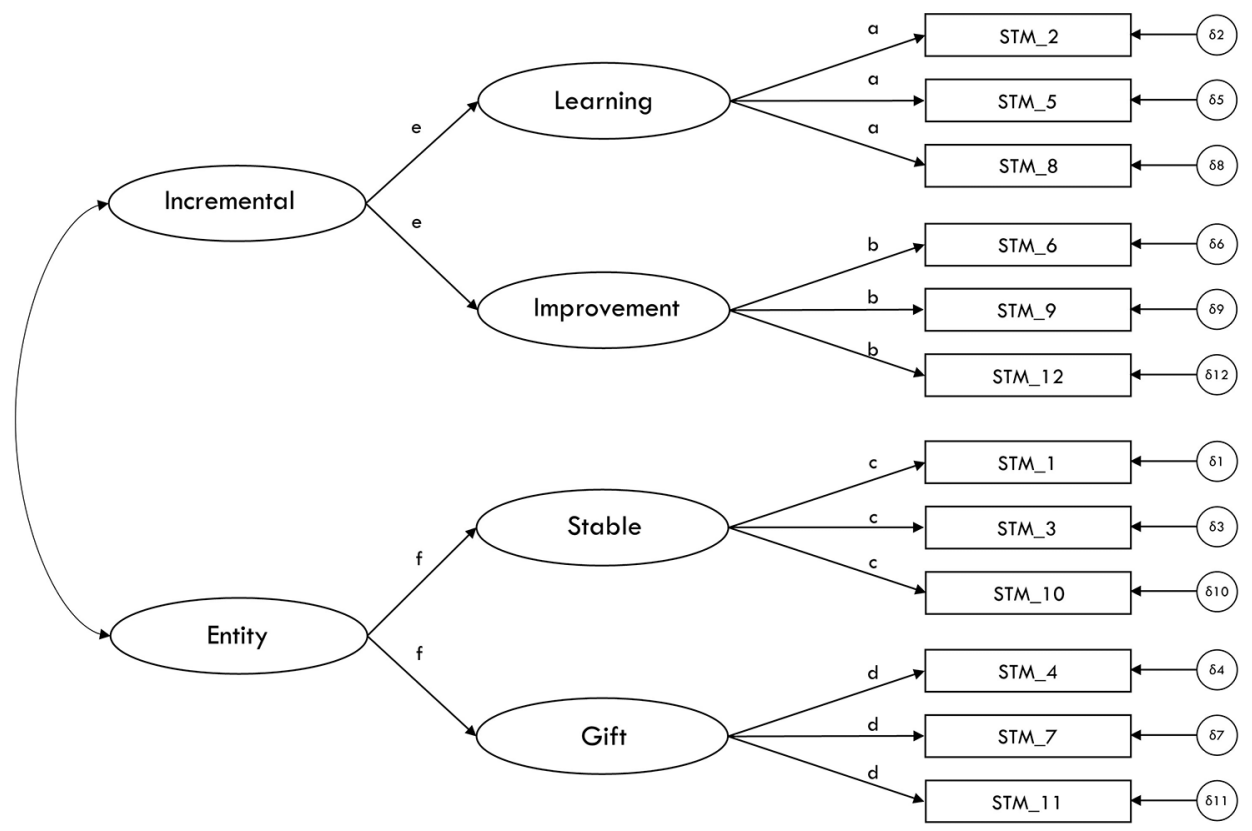

Anmerkung. STM_X = Item-Kennung. 
Modell 0 (siehe Abbildung 2) wurde in die Analyse miteinbezogen, um zu überprüfen, ob die Subjektiven Theorien über Musikalität basierend auf früheren Arbeiten von Dweck et al. (1995) zu Subjektiven Theorien im Allgemeinen (Dweck, 2000) eindimensional erfasst werden können. Modell 1 (siehe Abbildung 3) basiert auf Studien zu den Subjektiven Theorien, die zeigen, dass es möglich ist, dass Individuen sowohl eine dynamische als auch stabile Theorie über eine menschliche Eigenschaft haben können, wobei diese beiden Theorien in einem negativen Zusammenhang stehen (Sarrazin et al., 1996). Modell 2 (siehe Abbildung 4) gründet sich auf Theorien, die differenzieren zwischen der Vorstellung über die Veränderbarkeit (Improvement vs. Stable) und über die Ursache (Gift vs. Learning) menschlicher Eigenschaften. Diese Unterscheidung wurde in der Fragebogenentwicklung zu den Subjektiven Theorien über sportliche Fähigkeiten u. a. mit unterschiedlichen Vorstellungen begründet, die im Wissenschafts- und Alltagsverständnis über sportliche Fähigkeiten existieren (Biddle et al., 2003; Sarrazin et al., 1996). Analog dazu finden sich auch in den Alltags- und Wissenschaftsvorstellungen von Musikalität Theorien, die einerseits grundsätzlich die Möglichkeit einer Veränderung überhaupt, und andererseits die Ursache dieser möglichen Nicht-Veränderung bzw. Veränderung, wie z. B. ein bestimmtes Übeverhalten, Zufallsfaktoren oder auch angeborene Eigenschaften, betreffen (Austin et al., 2006; Gembris, 2018; Hemming, 2002). Modell 3 (siehe Abbildung 5) beruht auf der Vermutung, dass die gemeinsame Varianz zwischen Learning und Improvement bzw. zwischen Stable und Gift durch die beiden höher gestellten Faktoren Incremental und Gift erklärt werden kann (Biddle et al. 2003).

Im Kontext von Modellüberprüfungen wird kritisiert, dass in der Forschung oft freie Faktorenmodelle geschätzt werden, während in der Praxisverwendung die Skalenwerte mit einfachen Summen gebildet werden (McNeish \& Wolf, 2020). Deshalb wurde bei der Datenanalyse der vorliegenden Studie alle Messmodelle so eingeschränkt, dass immer die identischen Gewichte innerhalb eines Faktors addiert werden. Die so theoretisch angenommenen Messmodelle bieten damit eine interpretierbare Lösung für die Verwendung in der Praxis. Da in der Praxis üblicherweise ungewichtete Summen-Scores für die einzelnen Faktoren berechnet werden, ist zu erwarten, dass das in der Studie berechnete Modell durch die vorgenommenen Einschränkungen in der Datenanalyse auch für die Verwendung des Fragebogens in der Praxis gültig ist.

Der Modell-Fit von allen vier Modellen wurde mit folgenden Indizes überprüft: Comparative Fit Index (CFI), Tucker Lewis Index (TLI), Root Mean Square Error of Approximation (RMSEA), Standardized Root Mean Square Residual (SRMR), zusätzlich zu Informationen zum Chi-Quadrat-Test $\left(X^{2}\right)$ und den Freiheitsgraden ( $d f$ ). Für die ersten beiden Modell-Fit Indizes (CFI und TLI) zeigen Werte über ,90 einen guten Fit des Modells an, während gute Modelle einen RMSEA unter ,06 und einen SRMR unter ,08 benötigen (Hu \& Bentler, 1999). Um die Modelle miteinander vergleichen zu können, wurde das Bayesian Information Criterion (BIC) verwendet. Die standardisierten Fit-Werte der vier Messmodelle wurden anhand eines $X^{2}$-Differenztests miteinander verglichen.

Bei dem durch die KFA identifizierten Modell wurde danach zur Bestimmung der internen Konsistenz der einzelnen Teilskalen Reliabilitätsanalysen mittels Cronbachs Alpha durchgeführt (Fragestellung 2) und die Korrelationen zwischen den Items eines Faktors berechnet. Nach Cronbach (1951) sollten die $\alpha$-Werte über ,7 liegen. Des Weiteren wurde für beide Stichproben der Mittelwert, die Standardabweichung und die Schiefe jeder latenten Variablen berechnet. 


\section{Konfirmatorische Faktorenanalyse}

Für die englische und deutsche Stichprobe zeigt der Mardia's-Test, dass die vorliegenden Daten keiner multivariaten Normalverteilung folgen (GB: $X^{2}$ skew $=1349,96, p<, 001, D E: X^{2}$ skew $=940,67, p<, 001$ ). Demzufolge wurde für die nachfolgenden Analysen die robuste Maximum-Likelihood-Schätzung mit Full Information Maximum Likelihood (FIML) für die fehlenden Daten verwendet, da diese stabiler gegenüber nicht normalverteilten Daten ist (Hu et al., 1995).

Die umfassenden Fit Indizes aller untersuchter Modelle sind in Tabelle 1 dargestellt. Sowohl für den englischals auch deutschsprachigen Fragebogen zeigt die KFA insgesamt keine zufriedenstellenden Ergebnisse für M0, M1 und M2, während das hierarchische M3 eine akzeptable Übereinstimmung mit den empirischen Daten aufzeigt (M3 [GB]: $x^{2} / d f=2,87, \mathrm{CFI}=, 919, \mathrm{TLI}=, 909, \mathrm{RMSEA}=, 062, \mathrm{SRMR}=, 085, \mathrm{M} 3$ [DE]: $\mathrm{X}^{2} / d f=1,78$, $\mathrm{CFI}=, 899, \mathrm{TLI}=, 887, \mathrm{RMSEA}=, 051, \mathrm{SRMR}=, 066)$. Das Bayesian Information Criterion (BIC), welches einen direkten Vergleich der Modelle ermöglicht, zeigt in der englischen und deutschen Stichprobe jeweils den niedrigsten Wert für M3. Der Modellvergleich mittels eines $x^{2}$-Differenz-Tests zeigt, dass M3 sowohl in der deutschen als auch englischen Stichprobe eine statistisch signifikant $(p<, 001)$ bessere Anpassung als M0 $\left(\mathrm{GB}: \mathrm{X}^{2}(6)=707,29, p<, 001, \mathrm{DE}: \mathrm{X}^{2}(6)=258,99, p<, 001\right), \mathrm{M} 1\left(\mathrm{~GB}: \mathrm{X}^{2}(4)=128,73, p<, 001, \mathrm{DE}: \mathrm{X}^{2}(4)\right.$ $=65,64, p<, 001)$ und M2 (GB: $X^{2}(3)=233,31, p<, 001$, DE: $\left.X^{2}(3)=75,53, p<, 001\right)$ hat. Die Ergebnisse sprechen demnach für das Modell 3 , welches im Folgenden genauer beschrieben wird.

Tabelle 1

Fit Indices für die vier Modelle der KFA

\begin{tabular}{|c|c|c|c|c|c|c|c|c|c|}
\hline Stichprobe/Modell & $x^{2}$ & $d f$ & $x^{2} / d f$ & $p\left(x^{2}\right)$ & CFI & TLI & RMSEA & SRMR & BIC \\
\hline \multicolumn{10}{|l|}{ Englisch } \\
\hline MO & 1287,51 & 65 & 19,81 & $<, 001$ & ,087 & ,073 & , 196 & ,220 & 19070 \\
\hline M1 & 340,38 & 63 & 5,40 & $<, 001$ & ,796 & ,786 & ,094 & ,093 & 17922 \\
\hline M2 & 463,37 & 62 & 4,47 & $<, 001$ & ,711 & ,693 & ,113 & , 168 & 18066 \\
\hline M3 & 169,16 & 59 & 2,87 & $<, 001$ & ,919 & ,909 &, 062 & ,085 & 17744 \\
\hline \multicolumn{10}{|l|}{ Deutsch } \\
\hline Mo & 445,58 & 65 & 6,86 & $<, 001$ & ,234 & ,222 & , 135 & ,139 & 13185 \\
\hline M1 & 179,35 & 63 & 2,85 & $<, 001$ & ,761 & ,750 & ,077 & ,078 & 12878 \\
\hline M2 & 195,28 & 62 & 3,15 & $<, 001$ & ,729 & ,712 & ,082 & , 108 & 12903 \\
\hline M3 & 104,98 & 59 & 1,78 & $<, 001$ & ,899 & ,887 & ,051 & ,066 & 12815 \\
\hline
\end{tabular}

Anmerkung. $\mathrm{X}^{2}=$ Chi-Quadrat; $d f$ = Freiheitsgrade; CFI = Comparative Fit Index; TLI = Tucker Lewis Index; RMSEA = Root Mean Square Error of Approximation; SRMR = Standardized Root Mean Square Residual; BIC = Bayesian Information Criterion.

Die standardisierten Faktorenladungen der Faktoren erster Ordnung liegen in der englischen Stichprobe zwischen ,55 und ,79 (Abbildung 6) und in der deutschen Stichprobe zwischen ,51 und ,67 (Abbildung 7). Für die Faktoren zweiter Ordnung konnten standardisierte Faktorenladungen zwischen ,72 und ,94 (englische Stichprobe) und ,64 und ,85 (deutsche Stichprobe) berechnet werden. Die standardisierte Kovarianz zwischen dem Faktor Incremental und dem Faktor Entity in dem hierarchischen Modell liegt in der englischen Stichprobe bei $r=-, 57(p<, 001)$ und in der deutschen Stichprobe bei $r=-, 21(p<, 05)$. Die Faktorenladungen, Messfehlervarianzen der Indikatorvariablen und Varianzen der Faktoren erster Ordnung finden sich zudem in Tabelle A2 und A3 im Anhang. 


\section{Abbildung 6}

Modell 3 mit den standardisierten Faktorenladungen und den standardisierten Messfehlervarianzen der Indikatorvariablen für die englische Stichprobe

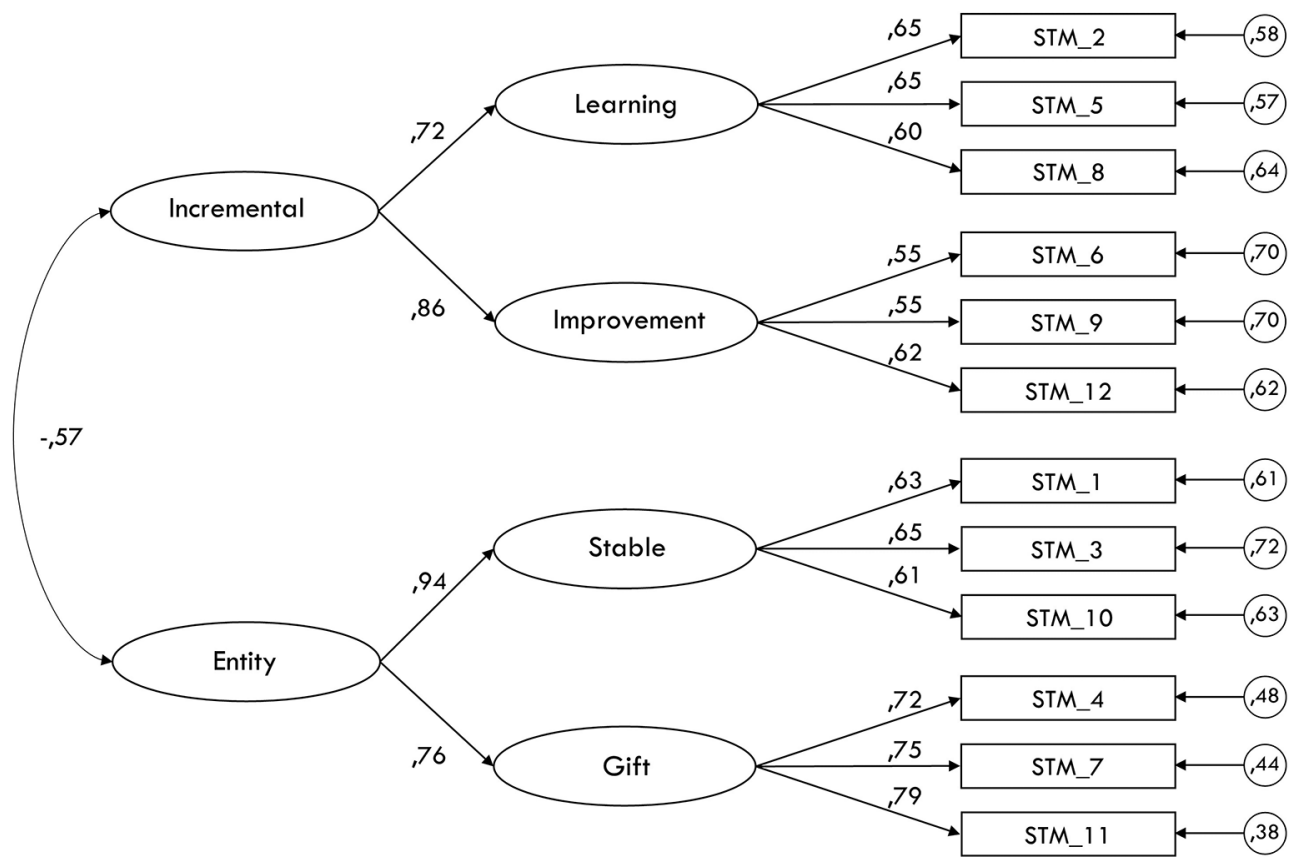

\section{Abbildung 7}

Modell 3 mit den standardisierten Faktorenladungen und den standardisierten Messfehlervarianzen der Indikatorvariablen für die deutsche Stichprobe

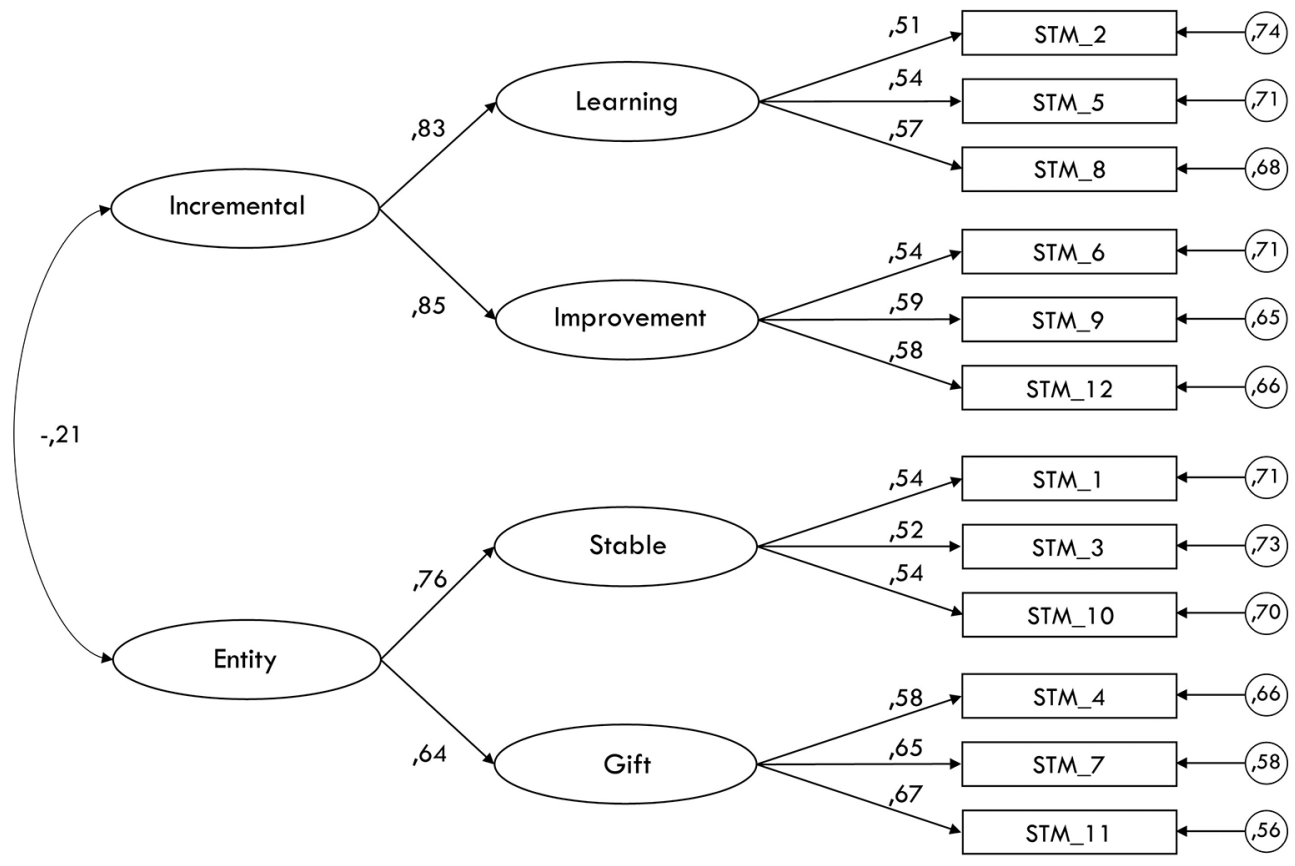




\section{Reliabilitätsanalysen und deskriptive Kennwerte}

Die Cronbachs Alpha Werte für die zwei Faktoren Incremental und Entity liegen in der englischen Stichprobe bei $\alpha=, 79$ und $\alpha=, 68$ und in der deutschen Stichprobe bei $\alpha=, 66$ und $\alpha=, 63$. Bezogen auf eine fünfstufige Likert-Skala von 1 = „strongly disagree“ bzw. „stimme überhaupt nicht zu“ bis 5 = „strongly agree“ bzw. „stimme sehr zu" hat der Faktor Incremental in der englischen bzw. deutschen Stichprobe ein arithmetisches Mittel (SD) von $M=4,10(0,52)$ bzw. $M=3,95(0,56)$ und der Faktor Entity von $M=2,52(0,71)$ bzw. $M=2,58(0,65)$. Die Items wurden dabei entsprechend der Modellannahmen bei der Berechnung nicht gewichtet miteinander verrechnet.

Während die Cronbachs Alpha Werte für die Subfaktoren Learning und Improvement in beiden Stichproben zwischen $\alpha=, 54$ und $\alpha=, 62$ liegen, haben die Subfaktoren Stable und Gift Cronbachs Alpha Werte zwischen $\alpha=, 55$ und $\alpha=, 79$. Für die Subfaktoren Learning und Improvement konnten Mittelwerte zwischen $M=3,83$ und $M=4,20$ berechnet werden und für die Subfaktoren Stable und Gift zwischen $M=2,44$ und $M=2,62$. Allgemein zeigten die Schüler*innen höhere Incremental Theorien als Entity Theorien. Der Cronbachs Alpha Wert, Mittelwert, die Standardabweichung und Schiefe für jeden Faktor des hierarchischen Modells 3 können der Tabelle 2 entnommen werden. Des Weiteren sind die Interkorrelationen der Faktoren Entity und Incremental für die englische und deutsche Stichprobe in Tabelle A4 bis A7 im Anhang dargestellt.

\section{Tabelle 2}

Ergebnisse der Reliabilitätsprüfung und deskriptive Statistik der englischen und deutschen Stichprobe für die latenten Faktoren erster und zweiter Ordnung des hierarchischen Modells 3

\begin{tabular}{|c|c|c|c|c|c|c|c|c|c|}
\hline \multirow[b]{2}{*}{ Faktor } & \multirow[b]{2}{*}{ Item-anzahl } & \multicolumn{4}{|c|}{ Englische Stichprobe } & \multicolumn{4}{|c|}{ Deutsche Stichprobe } \\
\hline & & $\alpha$ & $M$ & $S D$ & Schiefe & $\alpha$ & $M$ & $S D$ & Schiefe \\
\hline Incremental & 6 & ,79 & 4,10 & 0,52 & $-1,01$ & ,66 & 3,95 & 0,56 & $-0,79$ \\
\hline Entity & 6 & ,68 & 2,52 & 0,71 & 0,62 & ,63 & 2,58 & 0,65 & 0,11 \\
\hline Learning & 3 & ,62 & 4,01 & 0,64 & $-0,82$ &, 54 & 3,83 & 0,69 & $-0,73$ \\
\hline Improvement & 3 & ,62 & 4,20 & 0,64 & $-0,90$ &, 60 & 4,07 & 0,66 & $-0,79$ \\
\hline Stable & 3 & ,66 & 2,44 & 0,78 & 0,59 &, 55 & 2,62 & 0,79 & 0,07 \\
\hline Gift & 3 & ,79 & 2,60 & 0,86 & 0,40 & ,64 & 2,53 & 0,84 & 0,23 \\
\hline
\end{tabular}

Anmerkung. $\alpha$ = Cronbachs Alpha. Fünfstufige Likertskala (1 = „stimme überhaupt nicht zu“ bis 5 = „stimme sehr zu“).

\section{Diskussion und Ausblick}

Auf der Grundlage ihres Überblicks über Studien zu der Definition und Messung von Subjektiven Theorien fordern Lüftenegger und Chen (2017) weitere empirische Studien, die untersuchen, inwiefern die Subjektiven Theorien als ein- oder mehrdimensionales Konstrukt aufgefasst werden können. Die vorliegende Studie befasste sich mit diesem Bedarf und fokussierte dabei die Struktur der Subjektiven Theorien über Musikalität.

Die Ergebnisse der Analysen der angenommenen Modelle zeigen, dass der $x^{2}$-Test in beiden Stichproben für jedes Modell eine signifikante Abweichung zwischen den empirischen Daten zu dem jeweiligen Modell beschreibt. Dies kann jedoch auf die Größe der Stichprobe und der starken Einschränkung der Modelle aufgrund der gleichen Gewichtung der Faktorenladungen je Faktor zurückgeführt werden (Döring \& Bortz, 2016, S. 965). Da in beiden Stichproben die absoluten und relativen deskriptiven Gütekriterien akzeptable Werte für 
das Modell 3 aufzeigen und auch der $x^{2}$-Differenz-Test für dieses Modell spricht, zeigen die Analysen dieser Studie, dass die Subjektiven Theorien über Musikalität als ein multidimensionales, hierarchisches Konstrukt beschrieben werden können. Diese Struktur zeigt, dass, anstatt die Subjektiven Theorien eindimensional zu erfassen, ein differenzierter Blick auf das Konstrukt gelegt werden muss. Dieses Ergebnis entspricht den Modellvergleichen des Inventars von Biddle et al. (2003) zu Subjektiven Theorien über sportliche Fähigkeiten, das den Ausgangspunkt für die musikalische Adaption bildete.

Der Cronbachs Alpha Wert des Faktors Incremental der englischen Stichprobe liegt deutlich über dem Wert von $\alpha=, 7$, während der Faktor Entity in der englischen Stichprobe leicht unter diesem Wert liegt, genauso wie die beiden Faktoren zweiter Ordnung der deutschen Stichprobe. Die Cronbachs Alpha Werte der vier Subfaktoren liegen jedoch teilweise deutlich unter diesem Grenzwert von $\alpha=, 7$. Allerdings kritisieren mehrere Autor*innen (u. a. Cortina, 1993; Schmitt, 1996) die traditionellen Daumenwerte für die Interpretation von der Cronbachs Alpha und verweisen $u$. a. darauf, dass die Cronbachs Alpha Werte von der Anzahl der Items abhängig sind, d. h. Skalen mit nur wenigen Items generell niedrigere Alpha-Werte erreichen. Zudem fordert Schmitt (1996) vor allem eine aussagekräftige, theoretisch fundierte inhaltliche Begründung für die gebildeten Faktoren. Die Tatsache, dass die Subfaktoren des in dieser Studie verwendeten Fragebogens jeweils durch nur drei Items gebildet werden, könnte die Cronbachs Werte dieser Skalen erklären. Zudem werden in Studien zu dem Originalfragebogen zu den Subjektiven Theorien über sportliche Fähigkeiten gute Cronbachs Alpha Werte für die beiden Faktoren zweiter Ordnung berichtet (Biddle et al., 2003; Wang et al., 2009). Im Gegensatz zu der vorliegenden Studie testeten diese Autor*innen den Fragebogen jedoch mit Schüler*innen, die in Spanne und Durchschnitt älter sind als die Teilnehmenden der vorliegenden Studie. Da Studienergebnisse zeigen, dass die Struktur der Subjektiven Theorien sich im Laufe der weiterführenden Schule stark entwickelt und gerade jüngere Kinder noch nicht zwischen unterschiedlichen Aspekten der Subjektiven Theorien differenzieren können (Dweck, 2000), könnte auch die für die vorliegende Studie verwendete Stichprobe eine Ursache für die schlechteren Reliabilitätswerte sein. Der vorliegende Fragebogen zu den Subjektiven Theorien über Musikalität sollte demnach nochmals mit Schüler*innen validiert werden, die sich in der Mitte oder am Ende ihrer Schulzeit befinden, und auch in einer Stichprobe mit erwachsenen Personen überprüft werden. Bei einer Verwendung des Fragebogens sollten zudem die Messfehler anhand der hier ermittelten Reliabilitäten der jeweiligen Skalen berücksichtigt und die vier kurzen Skalen erster Ordnung nur mit Vorsicht individuell verwendet werden.

Die deskriptiven Analysen der vorliegenden Studie zu den Faktoren des hierarchischen Modells (M3) zeigen, dass die Faktoren, die eine stabile Subjektive Theorie beschreiben, eine rechtsschiefe Verteilung der Daten haben, während die Faktoren, die eine dynamische Subjektive Theorie beschreiben, stark linksschief gestreut sind. Diese hohen Zustimmungswerte zu den Skalen der dynamischen Subjektiven Theorie entsprechen vielen Ergebnissen weiterer Studien zu den Subjektiven Theorien. Auch unter Verwendung verschiedener Fragebögen sind die Daten gerade im Hinblick auf die dynamische Subjektive Theorie häufig sehr schief verteilt (Biddle et al., 2003; Wang et al., 2018).

Für die vorliegende Studie gelten weitere Einschränkungen, die Anlässe für zukünftige Forschung geben. Zum einen könnte die Validität des Konstrukts mithilfe von Berechnungen zur konvergenten und diskriminanten Validität stärker untersucht werden. Des Weiteren herrscht keine eindeutige Klarheit darüber, ob die Schüler*innen der englischen Stichprobe das Wort "You“ in den Items des Fragebogens dieser Studie tatsächlich als "Man“ oder als „Du“ verstehen. Zur Klärung könnten Kognitive Interviews mit Schüler*innen durchgeführt werden, um einen größeren Einblick in die kognitiven Prozesse der Teilnehmenden beim Beantworten des englischen 
Fragebogens zu bekommen (Prüfer \& Rexroth, 2005). Daran anschließend könnte in zukünftiger Forschung außerdem mit einem weiteren, neu entwickelten Fragebogen zu den Subjektiven Theorien über die eigene Musikalität die Annahme überprüft werden, dass die Subjektiven Theorien von Personen sich unterscheiden, je nachdem, ob es um die Veränderbarkeit der eigenen, persönlichen Eigenschaft oder der Eigenschaft im Allgemeinen geht (Dweck, 2000).

Eine weitere mögliche Weiterentwicklung des Fragebogens betrifft das verwendete Konzept von Musikalität. Die Musikpädagogik orientiert sich in ihrer Forschung zu Musikalität bzw. musikalischer Begabung stark an den Theorien und Modellen der Intelligenzforschung (Gembris, 2018). Deshalb liegt nahe, die Forschungen zu den Subjektiven Theorien über Intelligenz von Dweck (2000) auch auf Musikalität zu übertragen. Jedoch sind die Vorstellungen zur Struktur der Musikalität sehr unterschiedlich. Während manche von einem übergreifenden Generalfaktor ausgehen (u. a. Wing, 1981), nehmen andere an, dass es verschiedene Begabungsdimensionen gibt, die sehr unterschiedlich ausgeprägt sein können, aber in Wechselwirkung miteinander stehen ( $u$. a. Seashore et al., 1956). In den Items des Fragebogens der vorliegenden Studie wird nicht differenziert zwischen verschiedenen Begabungsdimensionen, sondern eine Vorstellung zu Musikalität als Generalfaktor erfragt. Musik wird außerdem in vielen verschiedenen Kontexten, wie z. B. außerhalb und innerhalb der Schule oder auch solistisch oder in Ensembles, gemacht. Obwohl es erste Arbeiten gibt, die Subjektive Theorien zu verschiedenen Dimensionen von Musikalität erfragen, z. B. zu einem Mindset of Singing Ability (Cogdill, 2013) oder auf theoretischer Ebene zu dem Growth Mindset in Choral Ensembles (Morrison, 2019), fehlen umfassende empirische Studien zu Subjektiven Theorien in verschiedenen musikalischen Kontexten oder Begabungsdimensionen.

Die vorliegende Studie stellt für solche zukünftige Forschung einen wichtigen Beitrag, da mit dem Messinstrument zur Erfassung der Subjektiven Theorien über Musikalität mit einer Unterscheidung nicht nur in eine stabile und dynamische Theorie, sondern durch die vier Faktoren erster Ordnung auch in den Ursprung und die Stabilität von Musikalität differenziert werden kann. Im Vergleich zu einem vierdimensionalen Modell (M2) ist das hierarchische Modell zudem immer noch sparsam und für die Verwendung des Messinstruments mit anderen Konstrukten gut geeignet. Das in dieser Studie validierte Testinstrument ermöglicht, individuelle Unterschiede in der dynamischen und stabilen Subjektiven Theorie über Musikalität von Schüler*innen zu identifizieren. Neben diesen individuellen Unterschieden können außerdem die Effekte von Interventionen auf die Subjektiven Theorien nachgewiesen werden. Gerade in der Forschung zu den Subjektiven Theorien über Intelligenz wurden in den letzten Jahren Interventionen entwickelt, die in großen empirischen Studien überprüft wurden und die eine positive Wirkung von einer hohen dynamischen Subjektiven Theorie auf schulische Leistungen nachweisen konnten (Yeager et al., 2019). In dem zu Beginn des Beitrags beschriebenen Modell der optimalen Motivation für Lernen in musikalischen Kontexten (Austin et al., 2006, S. 232) wird deutlich, dass neben den Self System, zu dem die Subjektiven Theorien über Musikalität gehören, das Social System einen großen Einfluss z. B. auf die musikalischen Aktivitäten und Leistungen von Schüler*innen hat. Dabei spielen gerade Lehrpersonen z. B. durch ihre Rückmeldungen im Musikunterricht eine große Rolle für die Ausbildung einer dynamischen Subjektiven Theorie bei Schüler*innen, wobei das Handeln und Denken der Lehrpersonen wiederum von deren eigenen Subjektiven Theorien über Musikalität gelenkt wird (Adams, 2019; O'Neill, 2011). Der in dieser Studie validierte Fragebogen kann auch dazu eingesetzt werden, um dieses Social System des Motivationsmodells besser zu verstehen. So könnte z. B. die Rolle von Lehrkräften als Vermittler*innen von einer dynamischen Subjektiven Theorien über Musikalität in Interventionsstudien untersucht werden. 
Das Motivationsmodell von Austin et al. (2006) ist ein Prozessmodell, d. h. dass die Beziehungen zwischen den vier beschriebenen Dimensionen dynamisch sind. Beispielsweise tendieren jüngere Kinder dazu, musikalische Fähigkeiten als etwas Veränderbares zu sehen, während sie diese mit der Zeit eher als etwas Stabiles und Unkontrollierbares wahrnehmen (Austin et al., 2006). In dem LongGold-Projekt, in dem die Studie dieses Beitrags eingebunden ist, soll diese Entwicklung der Subjektiven Theorien über Musikalität im Zusammenhang mit musikalischer und akademischer Leistung und musikalischer Aktivität über einen längeren Zeitraum untersucht werden. Die Ergebnisse der vorliegenden Studie bilden somit die Grundlage für die systematische Untersuchung der Bedingungsfaktoren von Subjektiven Theorien über Musikalität und deren Bedeutung für die individuelle Entfaltung musikalischen Potentials.

\section{Finanzierung}

Das Forschungsprojekt wird durch die Mittel der Alexander von Humboldt-Stiftung unterstützt.

\section{Interessenkonflikte}

Die Autorin erklärt, dass keine konkurrierenden Interessen bestehen.

\section{Danksagung}

Die Autorin ist für die Unterstützung dieser Veröffentlichung durch das LongGold-Projektteam dankbar.

\section{Ethikerklärung}

Die vorliegende Arbeit wurde in Übereinstimmung mit ethischen Prinzipien und Standards durchgeführt. Sie wurde von den Ethikkommissionen des Goldsmiths College, der Leibniz Universität Hannover und dem Ministerium für Kultus, Jugend und Sport Baden-Württemberg geprüft und genehmigt.

\section{Datenverfügbarkeit}

Alle erhobenen Forschungsdaten zu diesem Beitrag können aufgrund der Ethikvorgaben und aus datenschutzrechtlichen Gründen nicht öffentlich gemacht werden.

\section{Literatur}

Adams, K. (2019). Developing growth mindset in the ensemble rehearsal. Music Educators Journal, 105(4), 21-27. https://doi.org/10.1177/0027432119849473

Austin, J., Renwick, J., \& McPherson, G. E. (2006). Developing Motivation. In G. McPherson (Hrsg.), The child as musician: A handbook of musical development (S. 213-238). Oxford University Press.

Biddle, S. J. H., Wang, J., Chatzisarantis, N., \& Spray, C. (2003). Motivation for physical activity in young people: Entity and incremental beliefs about athletic ability. Journal of Sports Sciences, 21(12), 973-989. https://doi.org/10.1080/02640410310001641377

Burnette, J. L., O’Boyle, E. H., VanEpps, E. M., Pollack, J. M., \& Finkel, E. J. (2013). Mind-sets matter: A meta-analytic review of implicit theories and self-regulation. Psychological Bulletin, 139(3), 655-701. https://doi.org/10.1037/a0029531 
Cogdill, S. H. (2013). The identification of factors contributing to first-year college students' mindset of singing ability, and the relationship of that mindset to intent to participate in singing activities. Dissertation. University of Nebraska, Lincoln.

Connell, J. P. (1990). Context, self, and action: A motivational analysis of self-system processes across the life span. In D. Cicchetti (Hrsg.), The self in transition: Infancy to childhood (S. 61-97). University of Chicago Press.

Cortina, J. M. (1993). What is coefficient alpha? An examination of theory and applications. The Journal of Applied Psychology, 78(1), 98-104. https://doi.org/10.1037/0021-9010.78.1.98

Cronbach, L. J. (1951). Coefficient alpha and the internal structure of tests. Psychometrika, 16(3), 297-334. https://doi.org/10.1007/BF02310555

Döring, N., \& Bortz, J. (2016). Forschungsmethoden und Evaluation in den Sozial- und Humanwissenschaften (5. vollständig überarbeitete, aktualisierte und erweiterte Auflage). Springer.

Dweck, C. S., \& Grant, H. (2008). Self-theories, goals and meaning. In J. Y. Shah \& W. L. Gardner (Hrsg.), Handbook of Motivation Science (S. 405-416). Guilford Publications.

Dweck, C. (2008). Mindset: The new psychology of success. Ballantine Books.

Dweck, C. (2017). Selbstbild. Wie unser Denken Erfolge oder Niederlagen bewirkt. Piper.

Dweck, C. S. (1986). Motivational processes affecting learning. The American Psychologist, 41(10), 1040-1048. https://doi.org/10.1037/0003-066X.41.10.1040

Dweck, C. S. (2000). Self-theories: Their role in motivation, personality, and development (Essays in Social Psychology). Psychology Press.

Dweck, C. S., Chiu, C., \& Hong, Y. (1995). Implicit theories and their role in judgments and reactions: A world from two perspectives. Psychological Inquiry, 6(4), 267-285. https://doi.org/10.1207/s15327965pli0604_1

Gembris, H. (2018). Musikalität und Begabung. In M. Dartsch, J. Knigge, A. Niessen, F. Platz \& C. Stöger (Hrsg.), Handbuch Musikpädagogik. Grundlagen - Forschung - Diskurse (UTB, Bd. 5040, 1. Auflage, S. 235-245). Waxmann.

Hallam, S. (2006). Musicality. In G. McPherson (Hrsg.), The child as musician: A handbook of musical development (S. 93110). Oxford University Press.

Hargreaves, D. J., \& Lamont, A. (2017). The psychology of musical development. Cambridge University Press.

Helmke, A. (2017). Unterrichtsqualität und Lehrerprofessionalität. Diagnose, Evaluation und Verbesserung des Unterrichts (Schule weiterentwickeln, Unterricht verbessern, 7. Auflage). Kallmeyer.

Hemming, J. (2002). Begabung und Selbstkonzept. Eine qualitative Studie unter semiprofessionellen Musikern in Rock und Pop (Beiträge zur Musikpsychologie, Bd. 3). LIT.

Howe, M. J. A., Davidson, J. W., \& Sloboda, J. A. (1998). Innate talents: Reality or myth? Behavioral and Brain Sciences, 21(3), 399-407. https://doi.org/10.1017/S0140525X9800123X

Hu, L., \& Bentler, P. M. (1999). Cutoff criteria for fit indexes in covariance structure analysis: Conventional criteria versus new alternatives. Structural Equation Modeling, 6(1), 1-55. https://doi.org/10.1080/10705519909540118 
Hu, L.-T., Bentler, P. M., \& Hoyle, R. H. (1995). Evaluating model fit. In R. H. Hoyle (Hrsg.), Structural equation modelling: Concepts, issues, and applications (S. 76-99). Sage Publications.

Korkmaz, S., Goksuluk, D., \& Zararsiz, G. (2014). MVN: An R package for assessing multivariate normality. The R Journal, 6(2), 151-162. https://doi.org/10.32614/RJ-2014-031

Lüftenegger, M., \& Chen, J. A. (2017). Conceptual issues and assessment of implicit theories. Zeitschrift fur Psychologie mit Zeitschrift fur Angewandte Psychologie, 225(2), 99-106. https://doi.org/10.1027/2151-2604/a000286

MacNamara, Á., Holmes, P., \& Collins, D. (2006). The pathway to excellence: The role of psychological characteristics in negotiating the challenges of musical development. British Journal of Music Education, 23(3), 285-302.

https://doi.org/10.1017/S0265051706007066

McNeish, D., \& Wolf, M. G. (2020). Thinking twice about sum scores. Behavior Research Methods, 52(6), 2287-2305. https://doi.org/10.3758/s13428-020-01398-0

McPherson, G., \& Williamon, A. (2006). Giftedness and talent. In G. McPherson (Hrsg.), The child as musician: A handbook of musical development (S. 239-256). Oxford University Press.

Molden, D. C., \& Dweck, C. S. (2006). Finding "meaning" in psychology: A lay theories approach to self-regulation, social perception, and social development. The American Psychologist, 61(3), 192-203.

https://doi.org/10.1037/0003-066X.61.3.192

Morrison, S. (2019). The choral mindset: Supporting growth mindset in choral ensembles. Canadian Music Educator, 60(4), 36-40.

Müllensiefen, D., Harrison, P., Caprini, F., \& Fancourt, A. (2015). Investigating the importance of self-theories of intelligence and musicality for students' academic and musical achievement. Frontiers in Psychology, 6, Artikel 1702. https://doi.org/10.3389/fpsyg.2015.01702

O'Neill, S. A. (2011). Developing a young musician's growth mindset: The role of motivation, self-theories, and resiliency. In I. Deliège \& J. Davidson (Hrsg.), Music and the Mind: Essays in Honour of John Sloboda (S. 31-46). Oxford University Press.

O'Neill, S. A., \& Sloboda, J. A. (1997). The effects of failure on children's ability to perform a musical test. Psychology of Music, 25(1), 18-34. https://doi.org/10.1177/0305735697251003

Oxford University Press. (2020, December 29). Meaning of you in English. LEXICO. https://www.lexico.com/definition/you

R Core Team. (2020). R: A language and environment for statistical computing. R Foundation for Statistical Computing. Verfügbar am April 9, 2021 unter https://www.R-project.org/

Prinzie, P., \& Onghena, P. (2005). Cohort Sequential Design. In B. Everitt \& D. C. Howell (Eds.), Encyclopedia of Statistics in Behavioral Science (S. 319-332). John Wiley \& Sons.

Prüfer, P., \& Rexroth, M. (Eds.). (2005). Kognitive Interviews (GESIS-How-to: 15). Verfügbar am Dezember, 20, 2020 unter http://nbn-resolving.de/urn:nbn:de:0168-ssoar-201470

Rieche, H., Fischer, A. K., Geißler, C. \& Eitel, A. (2018). Wenn Schülerinnen und Schüler glauben, unmusikalisch zu sein: Erkennen angehende Musik-Lehrkräfte solche Überzeugungen? Beiträge empirischer Musikpädagogik, 9, 1-23. 
Rosseel, Y. (2012). Lavaan: An R package for structural equation modeling. Journal of Statistical Software, 48(2), 1-36. https://doi.org/10.18637/jss.v048.i02

Sarrazin, P., Biddle, S., Famose, J.-P., Cury, F., Fox, K., \& Durand, M. (1996). Goal orientations and conceptions of the nature of sport ability in children: A social cognitive approach. British Journal of Social Psychology, 35(3), 399-414. https://doi.org/10.1111/j.2044-8309.1996.tb01104.x

Schmitt, N. (1996). Uses and abuses of coefficient alpha. Psychological Assessment, 8(4), 350-353. https://doi.org/10.1037/1040-3590.8.4.350

Seashore, C. E., Lewis, D., \& Saetveit, J. G. (1956). Seashore measures of musical talents. The Psychological Corporation.

Sloboda, J., Davidson, J., \& Howe, M. J. A. (1994). Is everyone musical? The Psychologist, 7(8), 349-354.

Smith, B. P. (2005). Goal orientation, implicit theory of ability, and collegiate instrumental music practice. Psychology of Music, 33(1), 36-57. https://doi.org/10.1177/0305735605048013

Spinath, B. (2001). Implizite Theorien über die Veränderbarkeit von Intelligenz und Begabung als Bedingungen von Motivation und Leistung. Pabst.

Spinath, B., \& Schöne, C. (2003). Subjektive Überzeugungen bezüglich Bedingungen von Erfolg in Lern- und Leistungskontexten und deren Erfassung. In J. Stiensmeier-Pelster \& F. Rheinberg (Hrsg.), Diagnostik von Motivation und Selbstkonzept (Tests und Trends, Bd. 2, S. 15-27). Hogrefe.

Spychiger, M. (2013). Das musikalische Selbstkonzept. Wer ich bin und was ich kann in der Musik. Üben und Musizieren, 2013(6), 18-21.

Tempelaar, D., Rienties, B., Giesbers, B., \& Gijselaers, W. H. (2015). The pivotal role of effort beliefs in mediating implicit theories of intelligence and achievement goals and academic motivations. Social Psychology of Education, 18(1), 101-120. https://doi.org/10.1007/s11218-014-9281-7

Wang, C. K. J., Liu, W. C., Lochbaum, M. R., \& Stevenson, S. J. (2009). Sport ability beliefs, $2 \times 2$ achievement goals, and intrinsic motivation: The moderating role of perceived competence in sport and exercise. Research Quarterly for Exercise and Sport, 80(2), 303-312. https://doi.org/10.1080/02701367.2009.10599565

Wang, C. K. J., Tan, L., \& Dairianathan, E. I. (2018). Achievement goals, implicit theories, and intrinsic motivation: A test of domain specificity across music, visual art, and sports. Journal of Research in Music Education, 66(3), 320-337. https://doi.org/10.1177/0022429418784563

Wing, H. D. (1981). Standardised tests of musical intelligence. National Foundation for Educational Research.

Woolfolk, A. (2008). Pädagogische Psychologie. Pearson Studium.

Yeager, D. S., Hanselman, P., Walton, G. M., Murray, J. S., Crosnoe, R., Muller, C., Tipton, E., Schneider, B., Hulleman, C. S., Hinojosa, C. P., Paunesku, D., Romero, C., Flint, K., Roberts, A., Trott, J., lachan, R., Buontempo, J., Yang, S. M., Carvalho, C. M., ... Dweck, C. S. (2019). A national experiment reveals where a growth mindset improves achievement. Nature, 573, 364-369. https://doi.org/10.1038/s41586-019-1466-y

Yeager, D. S., \& Dweck, C. S. (2012). Mindsets that promote resilience: When students believe that personal characteristics can be developed. Educational Psychologist, 47(4), 302-314. https://doi.org/10.1080/00461520.2012.722805 


\section{Appendix}

\section{Tabelle A1}

Nummerierte Item-Liste des englischen und deutschen Fragebogens zur Erfassung der Subjektiven Theorien über Musikalität

\begin{tabular}{|c|c|c|}
\hline Item-Kennung & Englischer Fragebogen & Deutscher Fragebogen \\
\hline STM_1 & $\begin{array}{l}\text { You have a certain level of musical ability and you cannot } \\
\text { really do much to change it. }\end{array}$ & $\begin{array}{l}\text { Man hat ein gewisses Level an musikalischen Fähigkeiten } \\
\text { und kann nicht viel tun, um das zu ändern. }\end{array}$ \\
\hline STM_2 & $\begin{array}{l}\text { To be successful in music you need to learn and regularly } \\
\text { practice techniques and skills on your instrument. }\end{array}$ & $\begin{array}{l}\text { Um erfolgreich Musik zu machen, muss man regelmäßig } \\
\text { Techniken und Fertigkeiten an seinem Instrument lernen } \\
\text { und üben. }\end{array}$ \\
\hline STM_3 & $\begin{array}{l}\text { Even if you try, the level of musical ability you can reach } \\
\text { will change very little. }\end{array}$ & $\begin{array}{l}\text { Das erreichbare Level an musikalischen Fähigkeiten } \\
\text { verändert sich nur wenig, selbst wenn man es versucht. }\end{array}$ \\
\hline STM_4 & You need to have certain 'gifts' to be good at music. & $\begin{array}{l}\text { Man braucht eine bestimmte 'Begabung', um gut in Musik } \\
\text { zu sein. }\end{array}$ \\
\hline STM_5 & You need to learn and work hard to be good at music. & Man muss lernen und fleißig sein, um gut in Musik zu sein. \\
\hline STM_6 & You will always get better at music if you work hard at it. & $\begin{array}{l}\text { Man entwickelt seine musikalischen Fähigkeiten, wenn } \\
\text { man fleißig daran arbeitet. }\end{array}$ \\
\hline STM_7 & $\begin{array}{l}\text { To be good at music you need to be born with the qualities } \\
\text { which allow you success. }\end{array}$ & $\begin{array}{l}\text { Wenn man in Musik gut sein möchte, muss man mit den } \\
\text { Fähigkeiten geboren werden, die einen erfolgreich } \\
\text { machen. }\end{array}$ \\
\hline STM_8 & $\begin{array}{l}\text { You have to go through periods of learning and training to } \\
\text { reach a high level of performance in music. }\end{array}$ & $\begin{array}{l}\text { Um ein hohes Level an musikalischer Leistung zu } \\
\text { erreichen, muss man durch Phasen des Lernens und } \\
\text { Übens. }\end{array}$ \\
\hline STM_9 & $\begin{array}{l}\text { How good you are at music will always improve if you work } \\
\text { at it. }\end{array}$ & $\begin{array}{l}\text { Wie gut die eigenen musikalischen Fähigkeiten sind, } \\
\text { hängt immer davon ab, ob man daran arbeitet. }\end{array}$ \\
\hline STM_10 & It is difficult to change how good you are at music. & $\begin{array}{l}\text { Es lässt sich schwer ändern, ob man gut mit Musik } \\
\text { umgehen kann. }\end{array}$ \\
\hline STM_11 & You need to be naturally gifted to be good at music. & $\begin{array}{l}\text { Man muss eine natürliche Begabung haben, um gut in } \\
\text { Musik zu sein. }\end{array}$ \\
\hline STM_12 & $\begin{array}{l}\text { You will always get better at music if you put enough effort } \\
\text { into it. }\end{array}$ & $\begin{array}{l}\text { Man kann immer bessere musikalische Fähigkeiten } \\
\text { entwickeln, wenn man sich genug anstrengt. }\end{array}$ \\
\hline
\end{tabular}

Anmerkung. Fünfstufige Likert-Skala von 1 = „strongly disagree“ bzw. „stimme überhaupt nicht zu“ bis 5 = „strongly agree“ bzw. „stimme sehr zu“, der Fragebogen ist angelehnt an Biddle et al. (2003), die Übersetzung des englischen Fragebogens in die deutsche Sprache wurde in einem iterativen Verfahren von Projektmitarbeitenden des LongGold-Projekts durchgeführt und an einer kleinen Pilotstichprobe überprüft.

\section{Tabelle A2}

Standardisierte Faktorenladungen der manifesten Variablen der englischen und deutschen Stichprobe und die standardisierten Messfehlervarianzen der Indikatorvariablen für Modell 3

\begin{tabular}{|c|c|c|c|c|}
\hline \multirow[b]{2}{*}{ Faktor/Item-Kennung } & \multicolumn{2}{|c|}{ Englische Stichprobe } & \multicolumn{2}{|c|}{ Deutsche Stichprobe } \\
\hline & $\lambda$ & $0^{\delta}$ & $\lambda$ & $\theta^{\delta}$ \\
\hline \multicolumn{5}{|l|}{ Learning } \\
\hline STM_2 & ,65 &, 58 &, 51 & ,74 \\
\hline STM_5 &, 65 &, 57 &, 54 &, 71 \\
\hline STM_8 &, 60 & ,64 &, 57 & ,68 \\
\hline
\end{tabular}




\begin{tabular}{|c|c|c|c|c|}
\hline \multirow[b]{2}{*}{ Faktor/Item-Kennung } & \multicolumn{2}{|c|}{ Englische Stichprobe } & \multicolumn{2}{|c|}{ Deutsche Stichprobe } \\
\hline & $\lambda$ & $\theta^{\delta}$ & $\lambda$ & $0^{\delta}$ \\
\hline \multicolumn{5}{|l|}{ Improvement } \\
\hline STM_6 &, 55 & ,70 &, 54 & ,71 \\
\hline STM_9 &, 55 & ,70 &, 59 & ,65 \\
\hline STM_12 & ,62 & ,62 &, 58 & ,66 \\
\hline \multicolumn{5}{|l|}{ Stable } \\
\hline STM_1 & ,63 & 61 &, 54 & ,71 \\
\hline STM_3 & ,65 &, 57 &, 52 & ,73 \\
\hline STM_10 & ,61 & ,63 &, 54 & ,70 \\
\hline \multicolumn{5}{|l|}{ Gift } \\
\hline STM_4 &, 72 & ,48 &, 58 & ,66 \\
\hline STM_7 & ,75 & ,44 & 65 &, 58 \\
\hline STM_11 & ,79 & ,38 & 67 &, 56 \\
\hline
\end{tabular}

Anmerkung. $\lambda$ = Faktorenladung; $\Theta^{\bar{\delta}}=$ Messfehlervarianz der Indikatorvariable. Items siehe Tabelle A1 im Anhang.

\section{Tabelle A3}

Standardisierte Faktorenladungen der latenten Variablen erster Ordnung der englischen und deutschen Stichprobe und deren zugehörige standardisierten Varianzen für das Modell 3

\begin{tabular}{|c|c|c|c|c|}
\hline \multirow[b]{2}{*}{ Faktor 2. Ordnung/Faktor 1 . Ordnung } & \multicolumn{2}{|c|}{ Englische Stichprobe } & \multicolumn{2}{|c|}{ Deutsche Stichprobe } \\
\hline & $\lambda$ & $\varphi$ & $\lambda$ & $\varphi$ \\
\hline \multicolumn{5}{|l|}{ Incremental } \\
\hline Learning & ,72 & ,49 & ,83 & ,31 \\
\hline Improvement & ,86 &, 27 & ,85 & ,28 \\
\hline \multicolumn{5}{|l|}{ Entity } \\
\hline Stable & ,94 & ,13 & ,76 & ,42 \\
\hline Gift & ,76 & ,42 & ,64 & 60 \\
\hline
\end{tabular}

Anmerkung. $\lambda$ = Faktorenladung; $\varphi=$ Varianz der latenten Variablen.

\section{Tabelle A4}

Korrelationsmatrix der Items des Faktors Entity der englischen Stichprobe

\begin{tabular}{|c|c|c|c|c|c|c|}
\hline Item-Kennung/Korrelation & STM_4 & STM_7 & STM_11 & STM_1 & STM_3 & STM_10 \\
\hline \multicolumn{7}{|l|}{ STM_7 } \\
\hline$r$ & ,49 & & & & & \\
\hline$p$ & $<, 001$ & & & & & \\
\hline \multicolumn{7}{|l|}{ STM_11 } \\
\hline$r$ &, 56 & ,60 & & & & \\
\hline$p$ & $<, 001$ & $<, 001$ & & & & \\
\hline \multicolumn{7}{|l|}{ STM_1 } \\
\hline$r$ &, 30 & ,38 &, 40 & & & \\
\hline$p$ & $<, 001$ & $<, 001$ & $<, 001$ & & & \\
\hline \multicolumn{7}{|l|}{ STM_3 } \\
\hline$r$ & ,28 & ,33 & ,35 & ,49 & & \\
\hline$p$ & $<, 001$ & $<, 001$ & $<, 001$ & $<, 001$ & & \\
\hline
\end{tabular}




\begin{tabular}{|c|c|c|c|c|c|c|}
\hline Item-Kennung/Korrelation & STM_4 & STM_7 & STM_11 & STM_1 & STM_3 & STM_10 \\
\hline \multicolumn{7}{|l|}{ STM_10 } \\
\hline$r$ &, 27 & ,30 &, 35 & ,33 &, 37 & \\
\hline$p$ & $<, 001$ & $<, 001$ & $<, 001$ & $<, 001$ & $<, 001$ & \\
\hline
\end{tabular}

\section{Tabelle A5}

Korrelationsmatrix der Items des Faktors Entity der deutschen Stichprobe

\begin{tabular}{|c|c|c|c|c|c|c|}
\hline Item-Kennung/Korrelation & STM_4 & STM_7 & STM_11 & STM_1 & STM_3 & STM_10 \\
\hline \multicolumn{7}{|l|}{ STM_7 } \\
\hline$r$ & ,28 & & & & & \\
\hline$p$ & $<, 001$ & & & & & \\
\hline \multicolumn{7}{|l|}{ STM_11 } \\
\hline$r$ & ,37 & ,49 & & & & \\
\hline$p$ & $<, 001$ & $<, 001$ & & & & \\
\hline \multicolumn{7}{|l|}{ STM_1 } \\
\hline$r$ & 09 &, 22 & ,18 & & & \\
\hline$p$ & ,089 & $<, 001$ & $<, 001$ & & & \\
\hline \multicolumn{7}{|l|}{ STM_3 } \\
\hline$r$ & ,03 & ,18 & ,22 & ,34 & & \\
\hline$p$ &, 546 & $<, 001$ & $<, 001$ & $<, 001$ & & \\
\hline \multicolumn{7}{|l|}{ STM_10 } \\
\hline$r$ & ,04 & ,17 & ,23 & ,23 & ,30 & \\
\hline$p$ &, 438 & ,001 & $<, 001$ & $<, 001$ & $<, 001$ & \\
\hline
\end{tabular}

Anmerkung. Faktor Gift = STM_4, STM_7, STM_11; Faktor Stable = STM_1, STM_3, STM_10.

\section{Tabelle A6}

Korrelationsmatrix der Items des Faktors Incremental der englischen Stichprobe

\begin{tabular}{|c|c|c|c|c|c|c|}
\hline Item-Kennung/Korrelation & STM_6 & STM_9 & STM_12 & STM_2 & STM_5 & STM_8 \\
\hline \multicolumn{7}{|l|}{ STM_9 } \\
\hline$r$ & ,29 & & & & & \\
\hline$p$ & $<, 001$ & & & & & \\
\hline \multicolumn{7}{|l|}{ STM_12 } \\
\hline$r$ & ,39 & ,39 & & & & \\
\hline$p$ & $<, 001$ & $<, 001$ & & & & \\
\hline \multicolumn{7}{|l|}{ STM_2 } \\
\hline$r$ & ,22 & ,22 & ,24 & & & \\
\hline$p$ & $<, 001$ & $<, 001$ & $<, 001$ & & & \\
\hline \multicolumn{7}{|l|}{ STM_5 } \\
\hline$r$ & ,31 & ,23 &, 30 &, 43 & & \\
\hline$p$ & $<, 001$ & $<, 001$ & $<, 001$ & $<, 001$ & & \\
\hline \multicolumn{7}{|l|}{ STM_8 } \\
\hline$r$ & ,10 &, 08 &, 15 & ,27 &, 35 & \\
\hline$p$ & ,014 & ,048 & $<, 001$ & $<, 001$ & $<, 001$ & \\
\hline
\end{tabular}

Anmerkung. Faktor Improvement = STM_6, STM_9, STM_12; Faktor Learning = STM_2, STM_5, STM_8. 


\section{Tabelle A7}

Korrelationsmatrix der Items des Faktors Incremental der deutschen Stichprobe

\begin{tabular}{|c|c|c|c|c|c|c|}
\hline Item-Kennung/Korrelation & STM_6 & STM_9 & STM_12 & STM_2 & STM_5 & STM_8 \\
\hline \multicolumn{7}{|l|}{ STM_9 } \\
\hline$r$ & ,35 & & & & & \\
\hline$p$ & $<, 001$ & & & & & \\
\hline \multicolumn{7}{|l|}{ STM_12 } \\
\hline$r$ & ,28 & ,36 & & & & \\
\hline$p$ & $<, 001$ & $<, 001$ & & & & \\
\hline \multicolumn{7}{|l|}{ STM_2 } \\
\hline$r$ & ,08 &, 17 & ,22 & & & \\
\hline$p$ & , 102 & $<, 001$ & $<, 001$ & & & \\
\hline \multicolumn{7}{|l|}{ STM_5 } \\
\hline$r$ & ,22 & ,26 & ,32 & ,26 & & \\
\hline$p$ & $<, 001$ & $<, 001$ & $<, 001$ & $<, 001$ & & \\
\hline \multicolumn{7}{|l|}{ STM_8 } \\
\hline$r$ & ,20 & ,20 &, 24 & ,33 &, 26 & \\
\hline$p$ & $<, 001$ & $<, 001$ & $<, 001$ & $<, 001$ & $<, 001$ & \\
\hline
\end{tabular}

Anmerkung. Faktor Improvement = STM_6, STM_9, STM_12; Faktor Learning = STM_2, STM_5, STM_8. 